TITLE:

\title{
Dynamic van der Waals theory
}

$\operatorname{AUTHOR}(\mathrm{S}):$

Onuki, A

CITATION:

Onuki, A. Dynamic van der Waals theory. Physical Review E 2007, 75(3): 036304.

ISSUE DATE:

2007-03

URL:

http://hdl.handle.net/2433/49916

RIGHT:

Copyright 2007 American Physical Society 
PHYSICAL REVIEW E 75, 036304 (2007)

\title{
Dynamic van der Waals theory
}

\author{
Akira Onuki \\ Department of Physics, Kyoto University, Kyoto 606-8502, Japan
}

(Received 26 October 2006; published 9 March 2007)

\begin{abstract}
We present a dynamic van der Waals theory starting with entropy and energy functional with gradient contributions. The resultant hydrodynamic equations contain the stress arising from the density gradient. It provides a general scheme of two-phase hydrodynamics involving the gas-liquid transition in nonuniform temperature. Some complex hydrodynamic processes with evaporation and condensation are examined numerically. They are (i) adiabatically induced spinodal decomposition, (ii) piston effect with a bubble in liquid, (iii) temperature and velocity profiles around a droplet in heat flow, (iv) efficient latent heat transport at small liquid densities (the mechanism of heat pipes), (v) boiling in gravity with continuous bubble formation and rising, and (vi) spreading and evaporation of liquid on a heated boundary wall.
\end{abstract}

DOI: 10.1103/PhysRevE.75.036304

\section{INTRODUCTION}

The van der Waals theory provides a simple description of gas-liquid phase transitions in one-component fluids [1]. It is an equilibrium mean-field theory for hard sphere particles with long-range attractive interaction [2]. Moreover, in his pioneering paper in 1893, van der Waals introduced a gradient term in the Helmholtz free energy density to describe a gas-liquid interface. Such a gradient term began to be widely used in statistical mechanics of nonuniform states, since seminal papers by Ginzburg and Landau for type-I superconductors [3] and by Cahn and Hilliard for binary alloys [4].

In most phase transition theories, including those of dynamics, the temperature $T$ is a given parameter independent of space $[5,6]$. The Ginzburg-Landau theory is based on a free energy functional with homogeneous $T$. It goes without saying that there are a variety of situations, where phase transitions occur in inhomogeneous $T$ or in heat flow. In fluid systems, wetting dynamics [7-11], boiling processes $[12-14]$, and droplet motion $[15,16]$ are strongly influenced by applied heat flux. Rayleigh-Bénard convection is very complex in liquid crystals with first-order phase transition [17] and in binary fluid mixtures with phase separation [18-20]. In He4, the superfluid transition can be nonlinearly influenced by heat flux, where a sharp interface is produced separating superfluid and normal fluid [21,22]. Understanding such a problem is very difficult. We need to construct a dynamical model first to study nonequilibrium effects, where phase transitions and hydrodynamics are inseparably coupled.

In 1901, Korteweg proposed hydrodynamic equations for binary fluid mixtures including the stress induced by composition gradients [23]. Diffuse interfaces between two-phases then arise in solutions of the hydrodynamic equations in the presence of the gradient stress [24-26]. In this line, a number of two-dimensional simulations of diffuse-interface models have been presented to describe two-phase hydrodynamics in one-component fluids $[6,14]$ and in binary mixtures $[19,20,26-28]$. One-dimensional numerical solutions are themselves highly nontrivial in heat flow $[29,30]$. Note that diffuse-interface models or phase field models have been used in numerical analysis of dendrite instability in crystal growth [31-33]. As another line of research, critical dynamics in classical fluids was studied with inclusion of the gradient contributions of the mass density and the composition, which are relevant even in one-phase states with growing of the correlation length near the critical point $[5,6,34-38]$. The so-called model $\mathrm{H}$ for near-critical binary mixtures was originally devised to describe dynamics of the thermal fluctuations [5], but has also been used to describe phase separation processes [39] and steady states under shear flow $[6,40]$. We mention Kawasaki's hydrodynamic equations for van der Waals fluids with long-range interaction [36], where the stress is of a nonlocal convolution form but reduces to the well-known form in critical dynamics in the gradient approximation [41]. For $\mathrm{He} 4$, on the other hand, a set of nonlinear hydrodynamic equations with the gradient contributions was established in an early period [42], whose simplified version near the superfluid transition was later used in critical dynamics $[5,6]$ and in studying nonlinear effects of heat flow [21].

Recently, we presented a dynamic van der Waals theory on the basis of entropy and energy functionals containing the gradient contributions [15]. We then constructed hydrodynamic equations with the gradient stress and numerically solved them to examine droplet motion in heat flow. One of our findings is that the temperature becomes homogeneous within a droplet under applied heat flux without gravity. Latent heat transport is so efficient such that the interface region is nearly on the coexistence curve $T=T_{\mathrm{cx}}(p)$ even in nonequilibrium, where the pressure $p$ is uniform outside the droplet. This picture was confirmed in a subsequent hydrodynamic theory [16]. As a result, the Marangoni effect arising from inhomogeneous surface tension is not operative in one-component (pure) fluids, while it gives rise to much faster fluid and interface motions in mixtures even at very small impurity concentrations [43].

In this paper, we will present a general scheme of the dynamic van der Waals theory in one-component fluids in a general form and give numerical results of some fundamental but complicated processes with evaporation and condensation. Section II will be a theoretical part, while Sec. III will present some numerical illustrations of such processes. 


\section{THEORY}

\section{A. van der Waals theory}

We summarize the main results of the van der Waals theory $[1,6]$, which will be needed in constructing our theory. As a function of the number density $n$ and the temperature $T$, the Helmholtz free density $f(n, T)$ for monoatomic molecules is written as

$$
f=k_{B} \operatorname{Tn}\left[\ln \left(\lambda_{\mathrm{th}}^{d} n\right)-1-\ln \left(1-v_{0} n\right)\right]-\epsilon v_{0} n^{2},
$$

where $v_{0}=a^{d}$ is the molecular volume and $\epsilon$ is the magnitude of the attractive potential with $d$ being the space dimensionality. The molecular radius is given by $a=v_{0}^{1 / d}$. The $\lambda_{\text {th }}$ $=\hbar(2 \pi / m T)^{1 / 2}$ is the thermal de Broglie length with $m$ being the molecular mass. As functions of $n$ and $T$, the internal energy density $e$, the entropy $s$ per particle, and the pressure $p$ are given by [44]

$$
\begin{gathered}
e=d n k_{B} T / 2-\epsilon v_{0} n^{2}, \\
s=-k_{B} \ln \left(\lambda_{\mathrm{th}}^{d} n /\left(1-v_{0} n\right)\right]+k_{B}(d+2) / 2, \\
p=n k_{B} T /\left(1-v_{0} n\right)-\epsilon v_{0} n^{2} .
\end{gathered}
$$

In this paper, use will be made of the expressions for the sound velocity $c=\left[(\partial p / \partial n)_{s} / m\right]^{1 / 2}$ and the specific heat ratio $\gamma_{s}=C_{p} / C_{V}$

$$
\begin{gathered}
c=\left(k_{B} T / m\right)^{1 / 2}\left(1+2 / d-T_{s} / T\right)^{1 / 2}, \\
\gamma_{s}=1+2 / d\left(1-T_{s} / T\right) .
\end{gathered}
$$

where $C_{V}=d n k_{B} / 2$ is the constant-volume specific heat and $C_{p}$ is the isobaric specific heat per volume. We introduce the spinodal temperature,

$$
T_{s}=2 \epsilon v_{0} n\left(1-v_{0} n\right)^{2} / k_{B} .
$$

Maximization of $T_{s}(n)$ as a function of $n$ yields the critical temperature $T_{c}=8 \epsilon / 27 k_{B}$ and the critical density $n_{c}=1 / 3 v_{0}$.

As mentioned in Introduction, van der Waals introduced the gradient free energy density,

$$
f_{\text {gra }}=\frac{1}{2} M|\nabla n|^{2},
$$

We consider the equilibrium interface density profile $n$ $=n(x)$ varying along the $x$ axis. The chemical potential per particle $\mu(n, T)=(\partial f / \partial n)_{T}$ changes as

$$
\mu-\mu_{\mathrm{cx}}=\frac{M}{2} \frac{d^{2} n}{d x^{2}}+\frac{d}{d x} \frac{M}{2} \frac{d n}{d x},
$$

where $\mu_{\mathrm{cx}}(T)$ is the chemical potential on the coexistence curve. From $d p=n d \mu$ at constant $T$, the van der Waals pressure $p(n, T)$ in Eq. (2.4) is expressed as [6]

$$
p-p_{\mathrm{cx}}=\frac{M}{2} n \frac{d^{2} n}{d x^{2}}+\frac{d}{d x} \frac{M}{2} n \frac{d n}{d x}-M\left(\frac{d n}{d x}\right)^{2},
$$

where $p_{\mathrm{cx}}(T)$ is the the pressure on the coexistence curve. In Sec. II D, a stress tensor $\Pi_{i j}$ including gradient contributions will be introduced, in terms of which Eq. (2.10) is equivalent to $\Pi_{x x}=p_{\mathrm{cx}}$ [see discussions below Eq. (2.50)]. The surface tension $\gamma(T)$ is expressed as $[6,45,46]$

$$
\gamma=\int_{-\infty}^{\infty} d x M(d n / d x)^{2}
$$

We note that Eqs. (2.9)-(2.11) hold even if $M$ depends on $n$ as $M=M(n)$ (while $M$ was a constant in the original theory [1]).

\section{B. Gradient entropy and energy}

We generalize the van der Waals theory by including gradient contributions to the entropy and the internal energy as

$$
\begin{gathered}
\mathcal{S}_{b}=\int d \boldsymbol{r}\left[n s(n, e)-\frac{1}{2} C|\nabla n|^{2}\right], \\
\mathcal{E}_{b}=\int d \boldsymbol{r}\left[e+\frac{1}{2} K|\nabla n|^{2}\right],
\end{gathered}
$$

where the space integrals are within the container of the fluid. The entropy and energy contributions at the boundary walls will be introduced in Eq. (2.28) below. The entropy density and the internal energy density including the gradient contributions are thus written as

$$
\begin{gathered}
\hat{S}=n s-\frac{1}{2} C|\nabla n|^{2}, \\
\hat{e}=e+\frac{1}{2} K|\nabla n|^{2} .
\end{gathered}
$$

The gradient terms represent a decrease of the entropy and an increase of the energy due to inhomogeneity of $n$. They are particularly important in the interface region. For simplicity, we neglect the gradient terms proportional to $(\nabla n) \cdot \nabla e$ and $(\nabla e)^{2}$. The gravitational energy is also neglected, but its inclusion is trivial [see discussions around Eq. (2.51)]. In this work, in constructing a general theory, we allow that $C$ and $K$ depend on $n$ as

$$
C=C(n), \quad K=K(n) .
$$

However, in our simulations in the next section, we will set $K=0$ and assume that $C$ is independent of $n$.

We then define the local temperature $T=T(n, e)$ by

$$
\frac{1}{T}=\left(\frac{\delta}{\delta e} \mathcal{S}_{b}\right)_{n}=n\left(\frac{\partial s}{\partial e}\right)_{n},
$$

where $n$ is fixed in the derivatives. This definition of $T$ is analogous to that in a microcanonical ensemble. We will use it even for inhomogeneous $n$ and $e$ in nonequilibrium. We also define a generalized chemical potential $\hat{\mu}$ per particle including the gradient contributions by

$$
\hat{\mu}=-T\left(\frac{\delta \mathcal{S}_{b}}{\delta n}\right)_{\hat{e}}=\mu-T \nabla \cdot \frac{M}{T} \nabla n+\frac{M^{\prime}}{2}|\nabla n|^{2},
$$

where the internal energy density $\hat{e}$ in Eq. (2.15) is fixed so we set $\delta e=-\delta\left(K|\nabla n|^{2} / 2\right)$ in the functional derivative. The 
$\mu=(e+p) / n-T s$ is the usual chemical potential per particle and

$$
M=C T+K
$$

Obviously, $M$ is the coefficient of the gradient term in the Helmholtz free energy defined in equilibrium (see the next section). Under Eq. (2.16), $M=M(n, T)$ depends on $n$ and $T$, so $M^{\prime}=(\partial M / \partial n)_{T}$ in Eq. (2.18) is its derivative with respect to $n$. Now, regarding $\mathcal{S}_{b}$ as a functional of $n$ and $\hat{e}$, we consider small changes $\delta n$ and $\delta \hat{e}$ of $n$ and $\hat{e}$ which yield an incremental change of $\mathcal{S}_{b}$. Using Eq. (2.18) and $\delta \hat{e}=\delta e$ $-\delta\left(K|\nabla n|^{2} / 2\right)$, we obtain

$$
\delta \mathcal{S}_{b}=\int d \boldsymbol{r}\left(\frac{1}{T} \delta \hat{e}-\frac{\hat{\mu}}{T} \delta n\right)-\int d a \frac{M}{T}(\boldsymbol{\nu} \cdot \nabla n) \delta n .
$$

The second term is the surface integral, where $d a$ is the surface element and $\boldsymbol{\nu}$ is the outward normal unit vector at the surface.

We remark on the following two papers. Fixman proposed the energy and entropy functionals, but without the gradient entropy, to calculate the transport coefficients near the critical point [34]. Anderson et al. started with energy and entropy functionals to include the velocity field in the solidification problem [33].

\section{Equilibrium conditions}

In equilibrium, we maximize $\mathcal{S}_{b}$ at a fixed particle number $\mathcal{N}=\int d \boldsymbol{r} n$ and a fixed energy $\mathcal{E}_{b}=\int d \boldsymbol{r} \hat{\epsilon}$ for a fluid confined in a cell. First, we are interested in the bulk equilibrium. To this end, we introduce

$$
W=\mathcal{S}_{b} / k_{B}+\nu \mathcal{N}-\beta \mathcal{E}_{b},
$$

where $\nu$ and $\beta$ are the Lagrange multipliers. The maximization condition $(\delta W / \delta e)_{n}=0$ with respect to $e$ at fixed $n$ yields the homogeneity of the temperature,

$$
T=1 / k_{B} \beta
$$

and then

$$
W=\left(-\mathcal{F}+k_{B} T \nu \mathcal{N}\right) / k_{B} T
$$

becomes a functional of $n$. We introduce the Helmholtz free energy functional,

$$
\mathcal{F}=\mathcal{E}_{b}-T \mathcal{S}_{b}=\int d \boldsymbol{r}\left(f(n, T)+\frac{M}{2}|\nabla n|^{2}\right),
$$

where the integrand is $\hat{e}-T \hat{S}$, so $f=e-T n s$ and $M$ is defined by Eq. (2.19). In the Ginzburg-Landau theory, we furthermore minimize $W$ with respect to $n$ to obtain

$$
\hat{\mu}=k_{B} T \nu=\text { const, }
$$

where $\hat{\mu}$ is defined by Eq. (2.18). In the thermodynamic limit we find $W=p V / k_{B} T$ where $p$ is the pressure and $V$ is the total volume of the fluid.

In a two-phase state with a planar interface at $x \cong 0$, the surface tension $\gamma$ is expressed as Eq. (2.11). Here we introduce the grand potential density,

$$
g(n, T)=f(n, T)-\mu_{\mathrm{cx}} n+p_{\mathrm{cx}},
$$

where $\mu_{\mathrm{cx}}$ and $p_{\mathrm{cx}}$ are the values of $\mu$ and $p$ on the coexistence curve. It is nonvanishing in the interface region, where $n=n(x)$ changes from a gas density $n_{g}$ to a liquid density $n_{\ell}$ as a function of $x$. From Eq. (2.25) it holds the relation $g(n, T)=M(d n / d x)^{2} / 2$ and

$$
\gamma(T)=\int_{n_{g}}^{n_{\ell}} d n[2 M g(n, T)]^{1 / 2} .
$$

This expression holds even when $M$ depends on $n$ [6].

In real fluids we need to consider the boundary condition on the surface of the container. For simplicity, we consider the surface entropy and energy of the forms,

$$
\mathcal{S}_{s}=\int d a \sigma_{s}(n), \quad \mathcal{E}_{s}=\int d a e_{s}(n),
$$

where $d a$ is the surface element and the areal densities $\sigma_{s}$ and $e_{s}$ are assumed to depend only on the number density $n$ on the surface. We need to maximize the total entropy $\mathcal{S}_{\text {tot }}$ $=\mathcal{S}_{b}+\mathcal{S}_{s}$ under fixed $\mathcal{N}$ and $\mathcal{E}_{\text {tot }}=\mathcal{E}_{b}+\mathcal{E}_{s}$. To this end, we introduce

$$
W_{\text {tot }}=W+\int d a\left(\sigma_{s}-\beta e_{s}\right),
$$

where $\beta$ is common to that in Eq. (2.22). Maximization of the surface part of $W_{\text {tot }}$ additionally yields

$$
M \nu \cdot \nabla n+\left(\partial f_{s} / \partial n\right)_{T}=0,
$$

as the surface boundary condition. Here we use Eq. (2.20) and define the Helmholtz surface free energy density,

$$
f_{s}(n, T)=e_{s}-T \sigma_{s} .
$$

In equilibrium, the temperature is homogeneous and we should minimize the total Helmholtz free energy,

$$
\mathcal{F}_{\text {tot }}=\mathcal{F}+\int d a f_{s}(n, T),
$$

in order to calculate the surface density profile $[7,48]$. The surface free energy is usually expanded to second order in $n-n_{c}$ as

$$
f_{s}=-a_{s}\left(n-n_{c}\right)+b_{s}\left(n-n_{c}\right)^{2} / 2,
$$

where $a_{s}$ and $b_{s}$ are constants. Then the boundary condition (2.30) is written as

$$
M \nu \cdot \nabla n-a_{s}+b_{s}\left(n-n_{c}\right)=0
$$

\section{Generalized hydrodynamic equations}

We propose hydrodynamic equations taking account of the gradient entropy and energy. They are of the same forms as those of compressible fluids in the literature [47] except that the stress tensor contains gradient contributions. The guiding principle of their derivation is the non-negative definiteness of the entropy production rate in the bulk region. In the previous theories $[24,26,28]$ the gradient entropy was not 
assumed and $M=K$ in Eq. (2.19). We suppose that a fluid is in a solid container with controllable boundary temperatures and the velocity field $\boldsymbol{v}$ vanishes on the boundary. We hereafter include a gravity $g$ applied in the downward direction along the $z$ axis.

First, the number density $n$ obeys

$$
\frac{\partial n}{\partial t}=-\boldsymbol{\nabla} \cdot(n \boldsymbol{v}) .
$$

The mass density is defined by $\rho=m n$, where $m$ is the particle mass. Second, the momentum density $\rho \boldsymbol{v}$ obeys

$$
\frac{\partial}{\partial t} \rho \boldsymbol{v}=-\boldsymbol{\nabla} \cdot(\rho \boldsymbol{v} \boldsymbol{v})-\boldsymbol{\nabla} \cdot(\stackrel{\leftrightarrow}{\Pi}-\stackrel{\leftrightarrow}{\sigma})-\rho g \boldsymbol{e}_{z},
$$

where the last term arises from the gravity with $\boldsymbol{e}_{z}$ being the (upward) unit vector along the $z$ axis. The $\overleftrightarrow{\Pi}=\left\{\Pi_{i j}\right\}$ is the reversible stress tensor containing the gradient contributions, which is invariant with respect to time reversal $\boldsymbol{v} \rightarrow-\boldsymbol{v}$. The $\overleftrightarrow{\sigma}=\left\{\sigma_{i j}\right\}$ is the dissipative stress tensor of the form,

$$
\sigma_{i j}=\eta\left(\nabla_{i} v_{j}+\nabla_{j} v_{i}\right)+(\zeta-2 \eta / d) \delta_{i j} \boldsymbol{\nabla} \cdot \boldsymbol{v}
$$

with $\nabla_{i}=\partial / \partial x_{i}$. The $\eta$ and $\zeta$ are the shear and bulk viscosities, respectively. Third, including the kinetic part, we define the (total) energy density,

$$
e_{T}=\hat{e}+\rho v^{2} / 2 .
$$

We assume that the energy current is of the usual form in terms of the stress tensor $\overleftrightarrow{\Pi}-\overleftrightarrow{\sigma}$ and the thermal conductivity $\lambda$. Then $e_{T}$ is governed by [47]

$$
\frac{\partial}{\partial t} e_{T}=-\nabla \cdot\left[e_{T} \boldsymbol{v}+(\stackrel{\leftrightarrow}{\Pi}-\overleftrightarrow{\sigma}) \cdot \boldsymbol{v}\right]+\nabla \cdot(\lambda \nabla T)-g \rho v_{z} .
$$

Equivalently, we may rewrite the above energy equation in terms of the internal energy density $\hat{e}$ using Eqs. (2.35) and (2.36) as

$$
\frac{\partial}{\partial t} \hat{e}=-\boldsymbol{\nabla} \cdot(\hat{e} \boldsymbol{v})-\stackrel{\leftrightarrow}{\Pi}: \nabla v+\dot{\epsilon}_{v}+\nabla \cdot(\lambda \nabla T),
$$

where there is no term proportional to $g$. On the right-hand side of Eq. (2.40), the second term represents $-\Sigma_{i j} \Pi_{i j} \nabla_{i} v_{j}$ and $\dot{\epsilon}_{v}=\Sigma_{i j} \sigma_{i j} \nabla_{i} v_{j}$ is the viscous heat production rate. Use of Eq. (2.37) gives

$$
\dot{\epsilon}_{v}=\sum_{i j} \frac{\eta}{2}\left(\nabla_{i} v_{j}+\nabla_{j} v_{i}-\frac{2}{d} \delta_{i j} \boldsymbol{\nabla} \cdot \boldsymbol{v}\right)^{2}+\zeta(\boldsymbol{\nabla} \cdot \boldsymbol{v})^{2} \geqslant 0 .
$$

From Eq. (2.20) the time derivative of $\mathcal{S}_{b}$ becomes composed of bulk and surface contributions as

$$
\frac{d \mathcal{S}_{b}}{d t}=\int d \boldsymbol{r}\left(\frac{1}{T} \frac{\partial \hat{e}}{\partial t}-\frac{\hat{\mu}}{T} \frac{\partial n}{\partial t}\right)-\int d a(\boldsymbol{\nu} \cdot \nabla n) \frac{M}{T} \frac{\partial n}{\partial t} .
$$

From our hydrodynamic equations the first bulk term becomes

$$
\begin{aligned}
\left(\frac{d \mathcal{S}_{b}}{d t}\right)_{\text {bulk }}= & \int d \boldsymbol{r}\left[\boldsymbol{v} \cdot\left(\boldsymbol{\nabla} \cdot \frac{\stackrel{\leftrightarrow}{\Pi}}{T}+\hat{e} \boldsymbol{\nabla} \frac{1}{T}-n \boldsymbol{\nabla} \frac{\hat{\mu}}{T}\right)\right. \\
& \left.+\frac{1}{T}\left(\boldsymbol{\nabla} \cdot \lambda \boldsymbol{\nabla} T+\dot{\epsilon}_{v}\right)\right] .
\end{aligned}
$$

If there is no heat flow from outside, the above quantity should be non-negative definite. We thus require

$$
\sum_{j} \nabla_{j}\left(\frac{1}{T} \Pi_{i j}\right)=-\hat{e} \nabla_{i} \frac{1}{T}+n \nabla_{i} \frac{\hat{\mu}}{T} .
$$

Under Eq. (2.44) we obtain $\left(d \mathcal{S}_{b} / d t\right)_{\text {bulk }}=\int d \boldsymbol{r}\left(\dot{\epsilon}_{v}+\dot{\epsilon}_{\theta}\right) / T \geqslant 0$ without heat input from the boundary [47], where the thermal heat production rate is

$$
\dot{\epsilon}_{\theta}=\lambda(\nabla T)^{2} / T \geqslant 0 .
$$

Now we seek the symmetric tensor $\Pi_{i j}$ which satisfies Eq. (2.44). Notice the thermodynamic identity,

$$
d(p / T)=-e d(1 / T)+n d(\mu / T),
$$

following from the Gibbs-Duhem relation $d \mu=-s d T+d p / n$ [6], where $d(\cdots)$ denotes an infinitesimal change. If the gradient stress is neglected, we have $\Pi_{i j}=p \delta_{i j}$, as should be the case, where $p=p(n, T)$ is the van der Waals pressure in Eq. (2.4). If it is included, some calculations yield

$$
\Pi_{i j}=\left(p+p_{1}\right) \delta_{i j}+M\left(\nabla_{i} n\right)\left(\nabla_{j} n\right),
$$

where $p_{1}$ is a diagonal gradient part,

$$
\begin{aligned}
p_{1} & =\left(n M^{\prime}-M\right) \frac{|\nabla n|^{2}}{2}-M n \nabla^{2} n-T n(\nabla n) \cdot \nabla \frac{M}{T} \\
& =n \hat{\mu}-\hat{e}+T \hat{S}-p .
\end{aligned}
$$

In the first line of Eq. (2.48), the last term does not exist in the previous theories [26]. The second line indicates that Eq. (2.48) is a generalization of the thermodynamic identity $p$ $=n \mu-e-T n s$. In Appendix A, we will present another derivation of the above expression for $\overleftrightarrow{\Pi}$ using Eqs. (2.35) and (2.40). With the above form of $\overleftrightarrow{\Pi}$, the total entropy density $\hat{S}$ in Eq. (2.14) obeys

$$
\frac{\partial}{\partial t} \hat{S}=-\boldsymbol{\nabla} \cdot\left[\hat{S} \boldsymbol{v}-\frac{M}{T} n(\boldsymbol{\nabla} \cdot \boldsymbol{v}) \boldsymbol{\nabla} n-\frac{\lambda}{T} \boldsymbol{\nabla} T\right]+\left(\dot{\epsilon}_{v}+\dot{\epsilon}_{\theta}\right) / T .
$$

The additional entropy flux $-T^{-1} M n(\boldsymbol{\nabla} \cdot \boldsymbol{v}) \boldsymbol{\nabla} n$ is reversible and is nonexistent in the usual hydrodynamics [47]. The last term on the right-hand side is the usual entropy production rate per unit volume.

We make some further comments. (i) Gouin [24] and Anderson $e t$ al. [26] also started with the entropy production rate with $M=K=$ const [see Eq. (10) of Ref. [24] and Eq. (15) of Ref. [26]], but without assuming Eqs. (2.39) and (2.44) they included a reversible contribution (in our notation),

$$
\boldsymbol{J}_{\mathrm{GA}}=M(\boldsymbol{\nabla} \cdot \boldsymbol{v}) n \boldsymbol{\nabla} n,
$$

in the energy flux. We do not assume such a new energy flux in Eq. (2.39); instead, we have the last term $[\propto \nabla(M / T)]$ in 


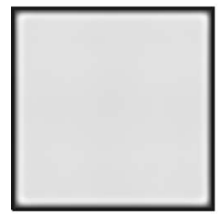

$t / t_{0}=50$

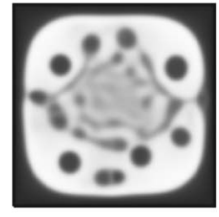

1500

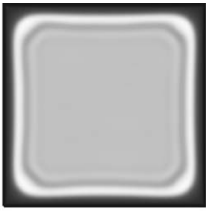

500

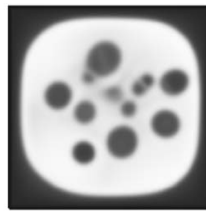

2000

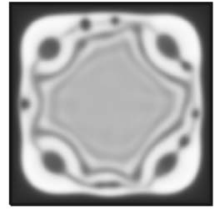

1000

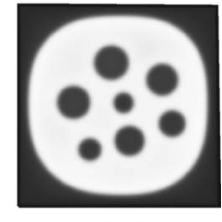

3200
FIG. 1. Density profiles in phase separation after lowering of the boundary temperature from $1.1 T_{c}$ to $0.91 T_{c}$ at $t=0$. Here the liquid layer (in black) at the boundary acts as a piston adiabatically expanding the interior.

$p_{1}$ in Eq. (2.48) and the additional entropy flux written as $T^{-1} J_{\mathrm{GA}}$ in Eq. (2.49) [49]. (ii) When $M$ and $T$ are constants independent of space, our expression for $\stackrel{\Pi}{T}$ reduces to that used near the critical point [6,37]. The off-diagonal components $M \nabla_{i} n \nabla_{j} n(i \neq j)$ give rise to the critical anomaly of the shear viscosity $[5,6,34]$ and the surface tension contribution to the shear viscosty in sheared two-phase states [40]. (iii) We consider the one-dimensional equilibrium case, where all the quantities vary along the $z$ axis. We then obtain $\boldsymbol{v}=\mathbf{0}, T$ $=$ const, and the equilibrium relation,

$$
\frac{d}{d z} \Pi_{z z}=-g \rho,
$$

which leads to Eq. (2.10) in two phase coexistence (by changing $z$ to $x$ and setting $g=0$ ). Using Eq. (2.44) we also obtain the equilibrium chemical potential,

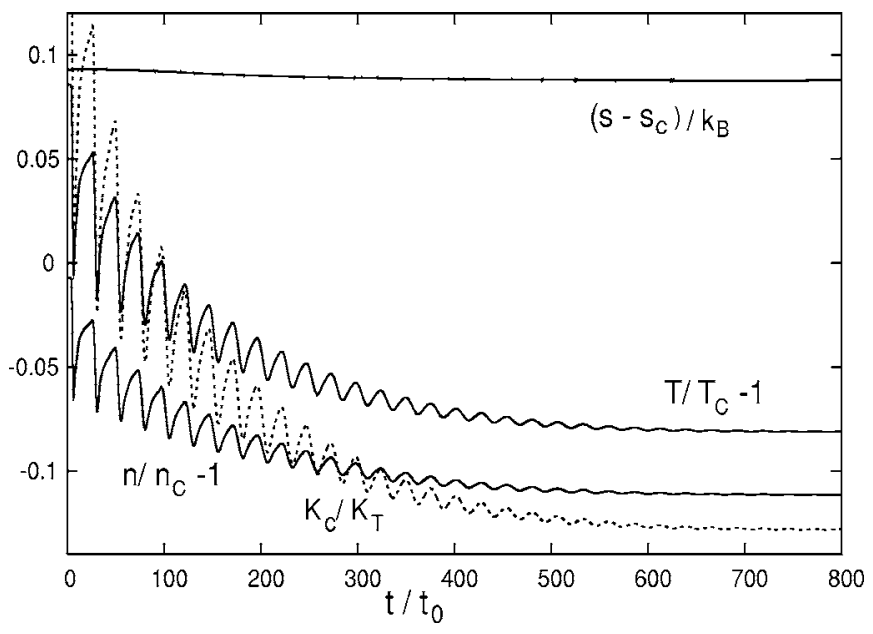

FIG. 2. Adiabatic time evolution of the temperature $T$ and the density $n$ at the cell center (lower solid lines) in the early stage after lowering of the boundary temperature from $1.1 T_{c}$ to $0.91 T_{c}$. Also shown is the inverse isothermal compressibility $1 / K_{T}$ multiplied by $K_{c}=n_{c} k_{B} T_{c}$ (dotted line). The entropy $s$ per particle (upper line) remains nearly unchanged, indicating that the process is adiabatic. The $s_{c}$ is the critical value. All the quantities are those at the cell center still without domains in this early stage.
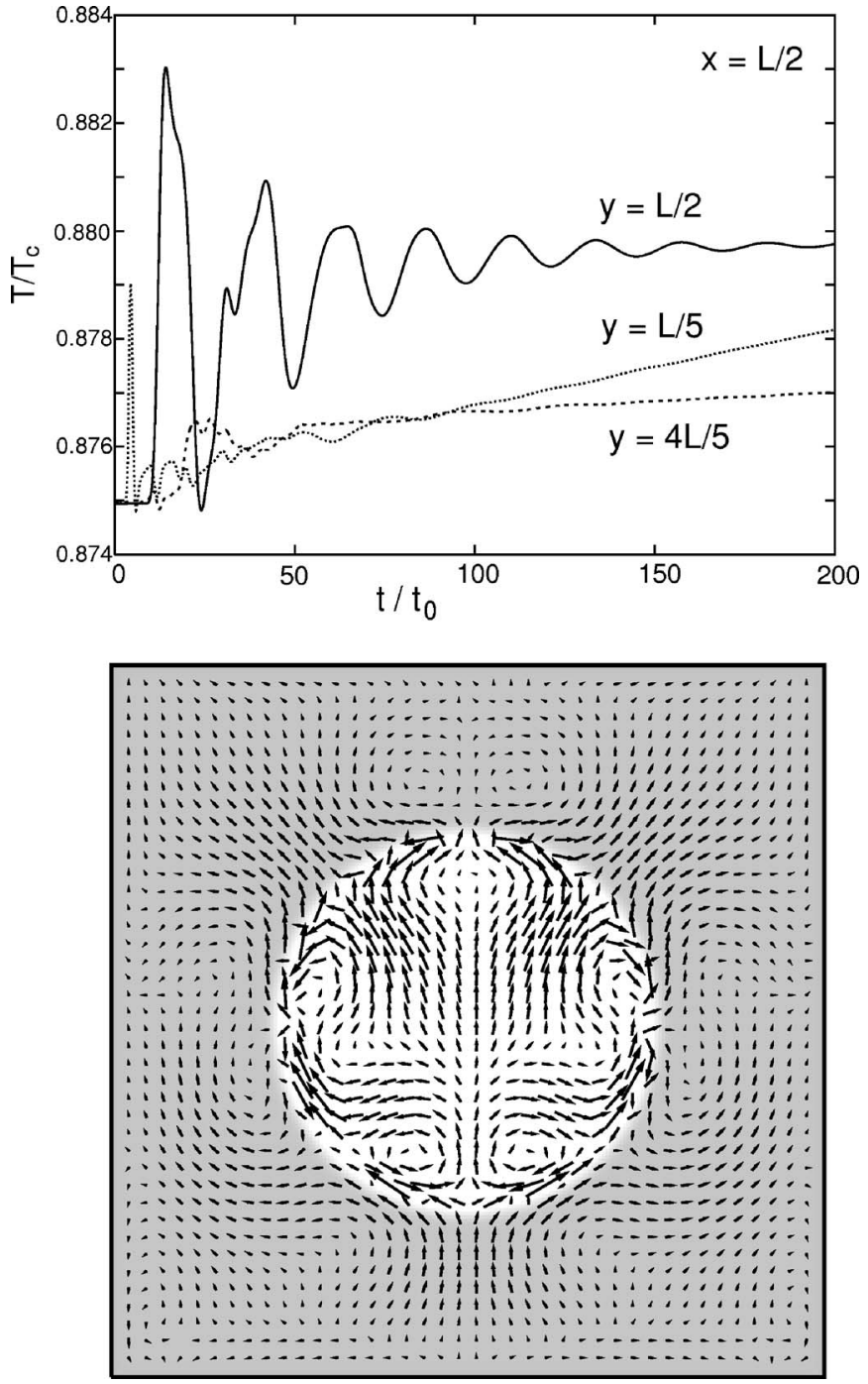

FIG. 3. Early stage time evolution due to the piston effect after a change of $T_{b} / T_{c}$ from 0.875 to 0.895 without gravity. A bubble with radius $50 \ell=L / 4$ is at the center. Upper plate: $T / T_{c}$ vs $t / t_{0}$ at the center $y=L / 2$ and in the liquid at $y=L / 5$ and $4 L / 5$, where $x$ $=L / 2$. Lower plate: $\boldsymbol{v}$ at $t / t_{0}=55$, where the gas region is strongly perturbed.

$$
\hat{\mu}+m g z=\text { const }
$$

which is a generalization of Eqs. (2.9) and (2.25) in gravity. In addition, we find $\Pi_{z z}-\Pi_{x x}=\Pi_{z z}-\Pi_{y y}=M(d n / d z)^{2}$. This is consistent with the surface stress tensor $\gamma\left(\nu_{i} \nu_{j}-\delta_{i j}\right) \delta_{s}$ in the thin interface limit [50], where $\boldsymbol{\nu}$ is the normal unit vector and $\delta_{s}$ is the $\delta$ function on the surface.

As the simplest boundary condition on the container surface, we may use the equilibrium condition (2.30) even in nonequilibrium and even when the temperature is not homogeneous on the boundary walls. This is justified when the equilibration at the boundary is much faster than that in the bulk. Then, under Eqs. (2.30) and (2.44) the total entropy $\mathcal{S}_{\text {tot }}=\mathcal{S}_{b}+\mathcal{S}_{s}$ changes in time as 


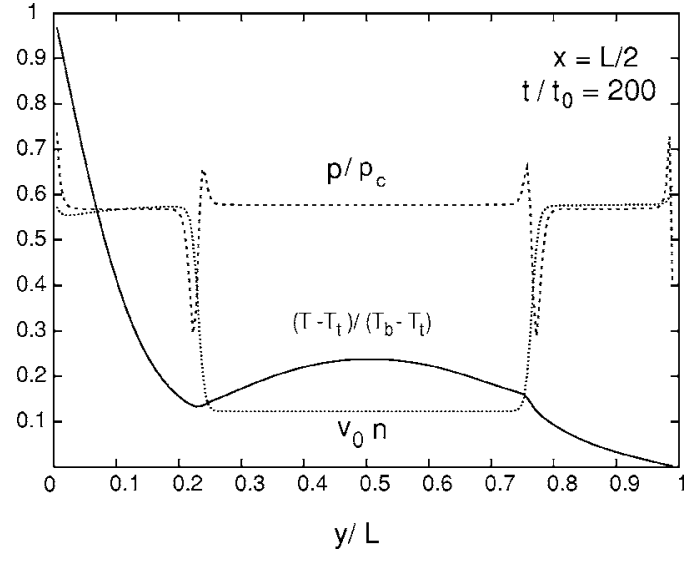

FIG. 4. $\left(T-T_{t}\right) /\left(T_{b}-T_{t}\right), p / p_{c}$, and $v_{0} n$ vs $y / L$ at $t / t_{0}=200$ and at $x=L / 2$, with $T_{b}=0.895 T_{c}$ and $T_{t}=0.875 T_{c}$, in the same run as in Fig. 3. The thermal diffusion layers are reaching at the bubble due to the small system size.

$$
\frac{d \mathcal{S}_{\mathrm{tot}}}{d t}=\int d \boldsymbol{r} \frac{\dot{\boldsymbol{\epsilon}}_{\theta}+\dot{\boldsymbol{\epsilon}}_{v}}{T}+\int d a \frac{\boldsymbol{\nu} \cdot \lambda \boldsymbol{\nabla} T+\dot{e}_{s}}{T},
$$

where $\dot{e}_{s}=\partial e_{s} / \partial t=\left(\partial e_{s} / \partial n\right) \partial n / \partial t$. On the right-hand side, the first term is the entropy production rate in the bulk, while the second term is due to the heat flux from outside and surface energy change. Note that the relation (2.53) holds even when the temperature is inhomogeneous on the boundary walls.

\section{NUMERICAL RESULTS}

\section{A. Method}

We integrated the hydrodynamic equations (2.35), (2.36), and (2.39) in two dimensions on a $202 \times 202$ lattice. We assume that $C$ in Eq. (2.12) is a constant independent of $n$ and $K$ in Eq. (2.13) vanishes. The mesh size of our simulation cell is chosen to be

$$
\ell=\left(C / 2 k_{B} v_{0}\right)^{1 / 2} \text {. }
$$

The interface thickness in two phase coexistence is of order $\ell$ far from the critical point and is given by $\left[8 T_{c} / 27\left(T_{c}\right.\right.$ $-T)]^{1 / 2} \ell$ close to the critical point. The system length is $L$ $=202 \ell$, so our system is very small. In the following figures, the $x$ axis is in the horizontal direction, while the $y$ axis is in the vertical direction, with $0 \leqslant x \leqslant L$ and $0 \leqslant y \leqslant L$. The velocity field $v$ is assumed to vanish on the walls; then, the average number density $\langle n\rangle=\int d \boldsymbol{r} n / V$ is a conserved quantity. Hereafter $\langle\cdots\rangle$ denotes taking the space average. As the boundary condition for the density, we assume Eq. (2.34) in the form

$$
2 \sqrt{2} \ell \boldsymbol{\nu} \cdot \nabla n=n-5 n_{c} / 2,
$$

on all the walls. This yields $a_{s}=\ell k_{B} T / 2 \sqrt{2}$ and $b_{s}=2 v_{0} a_{s}$ in Eq. (2.33). Then the boundary walls are wetted by liquid in equilibrium. The boundary condition for the temperature is as follows. In Figs. 1 and 2, we assume a common boundary temperature on all the walls. In Figs. 3-15, the bottom boundary temperature $T_{b}$ at $y=0$ and the top boundary tem-
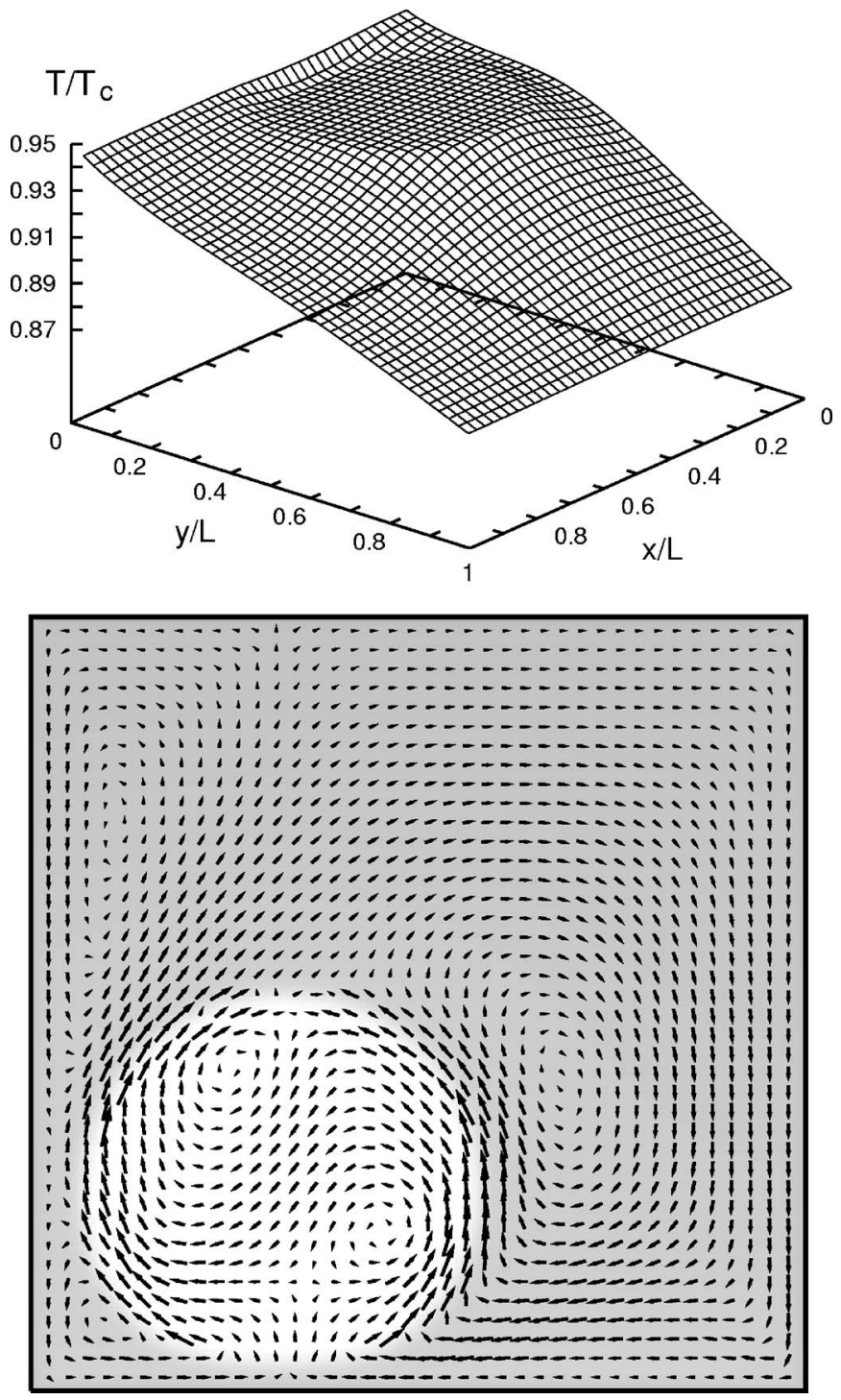

FIG. 5. $T$ (upper plate) and $\boldsymbol{v}$ and $n$ (lower plate) in a steady heat-conducting state at $T_{b}=0.945 T_{c}$ and $T_{t}=0.875 T_{c}$ under zero gravity. A bubble is attracted to the warmer boundary wall. Temperature homogeneity inside the bubble is attained by latent heat convection. The horizontal direction is along the $x$ axis in the text.

perature $T_{t}$ at $y=L$ will be taken to be different, while the side walls are thermally insulating or $\boldsymbol{\nu} \nabla T=0$ at $x=0$ and $L$. We use the words "top" and "bottom" even in the absence of gravity.

The transport coefficients strongly depend on the density $[30,51]$. That is, the shear viscosity and the thermal conductivity of liquid are usually much larger than those in gas except in the vicinity of the critical point. For simplicity, we assume them to be proportional to $n$ as

$$
\begin{gathered}
\eta=\zeta=\nu_{0} m n, \\
\lambda=k_{B} \nu_{0} n .
\end{gathered}
$$

Here the kinematic viscosity $\nu_{0}=\eta / \rho$ is assumed to be independent of $n$. For example, the liquid and gas densities are $n_{\ell}=1.70 n_{c}$ and $n_{g}=0.27 n_{c}$ in two phase coexistence at $T$ 

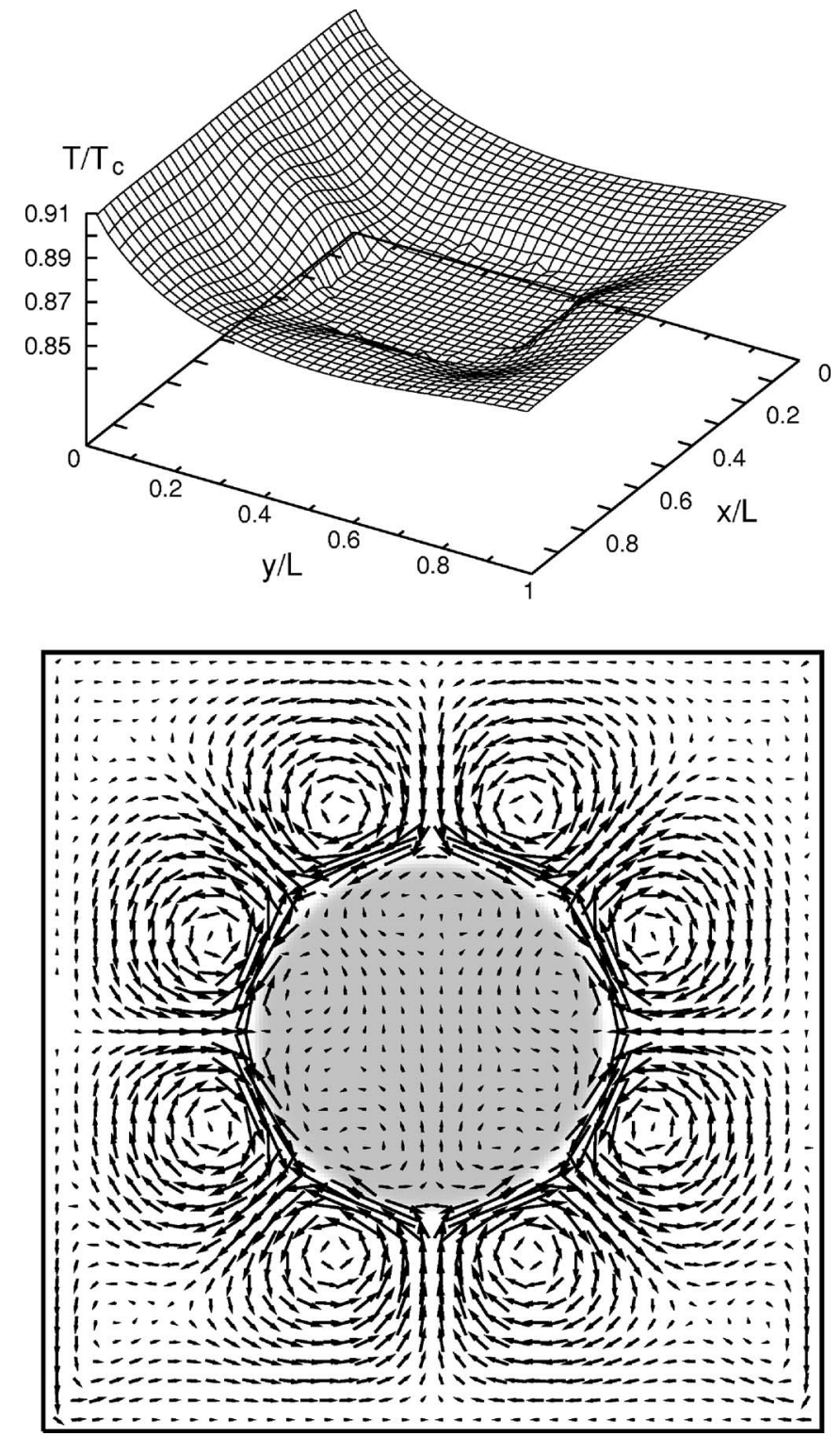

FIG. 6. $T$ (upper plate) and $\boldsymbol{v}$ and $n$ (lower plate) around a cool liquid droplet at $t=4565 t_{0}$ with $T_{b}=0.91 T_{c}$ and $T_{t}=0.875 T_{c}$ under zero gravity. Condensation is taking place at the interface and velocity field is induced.

$=0.875 T_{c}$ in the van der Waals model. Thus, under Eqs. (3.3) and (3.4) in the van der Waals model, the transport coefficients in liquid are larger than those in gas by $1.70 / 0 / 27$ $=6.3$ at $T=0.875 T_{c}$. Not close to the critical point, the thermal diffusion constant $D_{T}=\lambda / C_{p}$ is of order $\nu_{0}$, where the isobaric specific heat $C_{p}$ per unit volume is of order $k_{B} n$. Therefore, the Prandtl number $\operatorname{Pr}=\eta / \rho D_{T}$ is of order unity both in gas and liquid far from the critical point under Eqs. (3.3) and (3.4).

We will measure time in units of $t_{0}$ defined by

$$
t_{0}=\ell^{2} / \nu_{0}
$$

which is the viscous relaxation time $(\sim$ thermal diffusion time for $\operatorname{Pr} \sim 1$ ) on the scale of $\ell$. See Appendix B, where we will rewrite our hydrodynamic equations in dimensionless

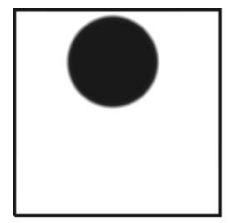

a) $t / t_{0}=15820$

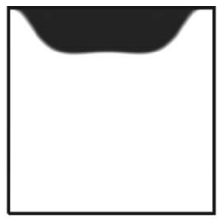

b) 16240

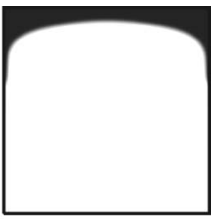

c) 16440
FIG. 7. Density profiles after that in Fig. 6. Illustrated are slow migration and rapid spreading after collision with the upper boundary.

forms. We then obtain a dimensionless number,

$$
\sigma=\nu_{0}^{2} m / \epsilon \ell^{2}=m \ell^{2} / \epsilon t_{0}^{2} .
$$

For $\mathrm{He}^{3}$ near the gas-liquid critical point with $T_{c}=3.32 \mathrm{~K}$, we have $\sigma^{1 / 2} \ell=\nu_{0}(\mathrm{~m} / \epsilon)^{1 / 2}=2.03 \times 10^{-8} \mathrm{~cm}$ by setting $\epsilon$ $=27 k_{B} T_{c} / 8$. In this paper we set $\sigma=0.01$ [see the sentences below Eq. (3.9) below for its reason]. The normalized gravity acceleration reads

$$
g^{*}=m g \ell / \epsilon,
$$

which is the gravity energy of a molecule over the distance $l$ divided by the van der Waals energy $\epsilon$. For $\mathrm{He}^{3}$ we have $g^{*}=3.2 \times 10^{-6} \ell$ with $\ell$ in units of $\mathrm{cm}$ under the earth gravity $g=0.98 \times 10^{3} \mathrm{~cm}^{3} / \mathrm{s}$. Thus $g^{*}$ is extremely small unless $\ell$ is large, which means that the gravity can be relevant only on macroscopic scales. In Figs. 9-15 below, however, we will set $g^{*}=10^{-4}$ to induce appreciable gravity effects even for our small system size.

In terms of $\sigma$ we express the sound velocity $c$ as

$$
c=A_{s} \sigma^{-1 / 2} \ell / t_{0},
$$

where $A_{s}$ is of order unity since Eq. (2.5) gives

$$
A_{s}=\left[k_{B}\left(2 T-T_{s}\right) / \epsilon\right]^{1 / 2} /\left(1-v_{0} n\right)
$$

in two dimensions. Therefore, the acoustic oscillation becomes apparent for small $\sigma$, but we require that the acoustic traversal time $L / c$ should be much longer than the microscopic time $t_{0}$ even for our small system length $L$ to obtain $\sigma^{1 / 2} L / \ell \gg 1$. A plane wave sound with small wave number $q$, evolves in time as $\exp \left(\Omega_{q} t\right)$ with $\Omega_{q}= \pm i c q-\Gamma_{s} q^{2} / 2$ in a homogeneous one-phase state. The sound damping coefficient $\Gamma_{s}$ is written as $\Gamma_{s}=[\zeta+(2-2 / d) \eta] / \rho+D_{T}\left(\gamma_{s}-1\right)$ [6]. If we use Eqs. (3.3) and (3.4), we find

$$
t_{0} \Omega_{q}= \pm i A_{s} \sigma^{-1 / 2} Q-\left(2-1 / \gamma_{s}\right) Q^{2},
$$

where $Q=\ell q$ is the scaled wave number. Therefore, in our simulation, the damping takes place on the time scale $t_{0} / Q^{2}$ and the acoustic oscillation can be seen for $Q \ll \sigma^{-1 / 2}$. Furthermore, the capillary wave frequency $\omega_{q}\left[\sim\left(\gamma q^{3} / m n_{\ell}\right)^{1 / 2}\right]$ in two phase states behaves as [46]

$$
t_{0} \omega_{q} \sim\left(1-T / T_{c}\right)^{3 / 4} \sigma^{-1 / 2} Q^{3 / 2} .
$$

The right-hand side gives the deformation rate of a droplet due to the surface tension if $Q=q \ell \sim 2 \pi \ell / R$ with $R$ being the droplet radius. 

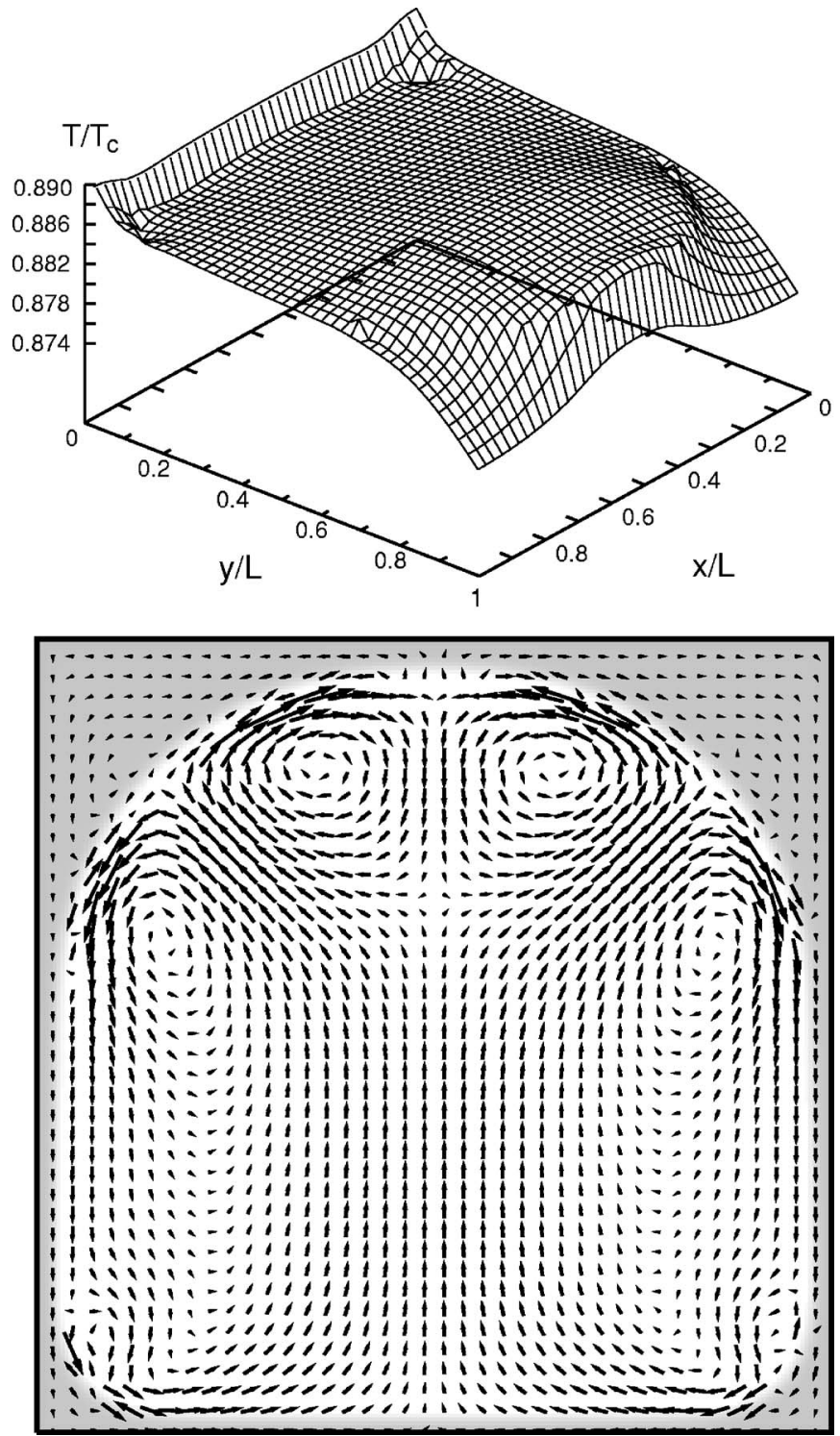

FIG. 8. $T$ (upper plate) and $\boldsymbol{v}$ and $n$ (lower plate) in a steady heat-conducting state at $T_{b}=0.89 T_{c}$ and $T_{t}=0.875 T_{c}$ under zero gravity.

\section{B. Phase separation by adiabatic expansion at $g=0$}

First, we investigate phase separation induced by the piston effect [52] taking place upon lowering of the boundary temperature. In such situations, due to contraction of thermal boundary layers, the interior can be expanded into metastable or unstable states adiabatically [6]. The piston effect is intensified near the critical point owing to the divergence of isobaric thermal expansion. With this method in near-critical one-component fluids, nucleation experiments were performed by Moldover et al. [53], while spinodal decomposition experiments were realized in space by Beysens et al. [54]. Recently Miura et al. [55] measured adiabatic density changes of order $10^{-7} n_{c}$ in the interior caused by sound pulses in supercritical states. In this paper, however, we present an example of adiabatic spinodal decomposition far below the critical point caused by the growth of a wetting layer.
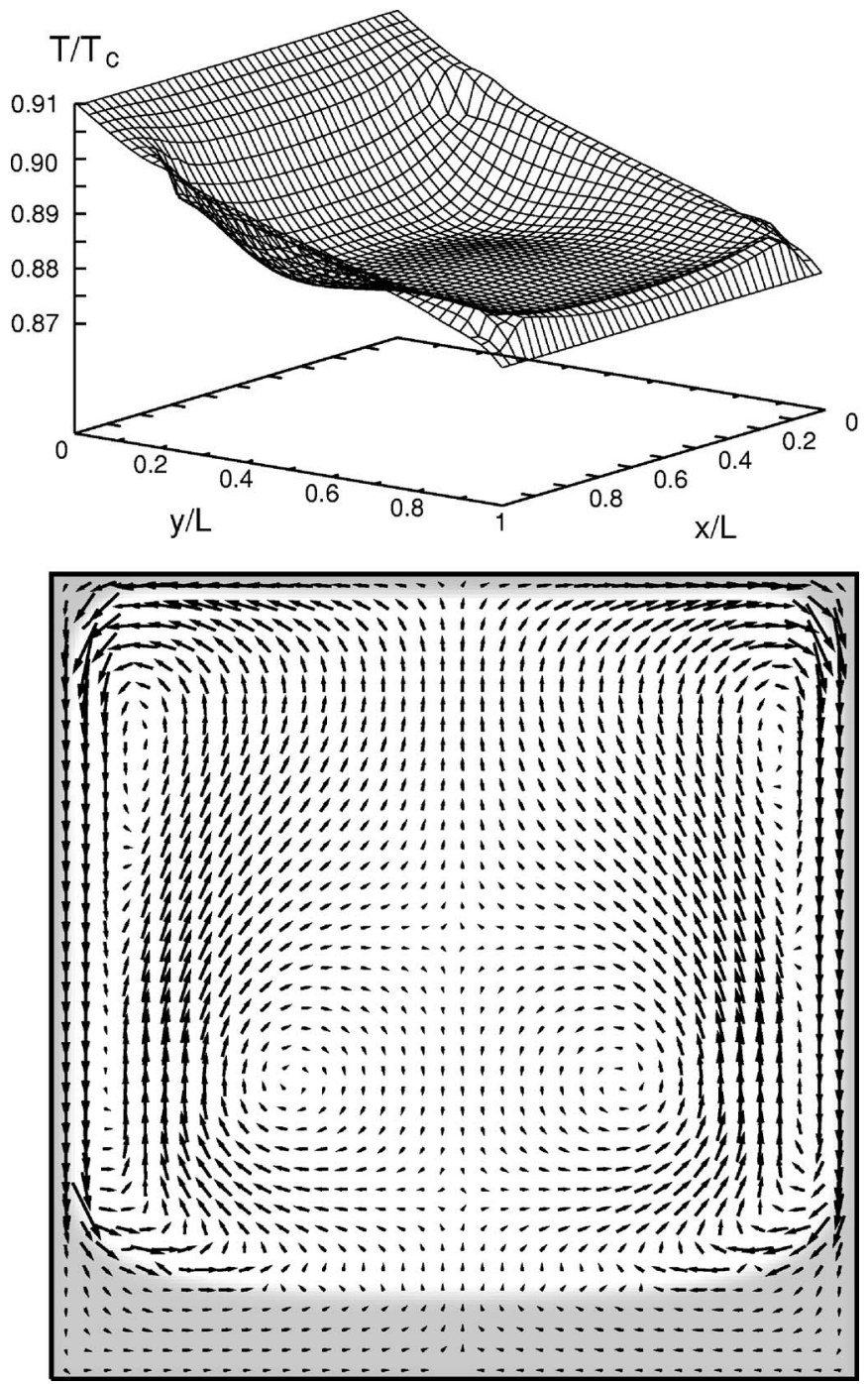

FIG. 9. $T$ (upper plate) and $\boldsymbol{v}$ and $n$ (lower plate) in a steady heat-conducting state at $T_{b}=0.91 T_{c}$ and $T_{t}=0.875 T_{c}$ under $g^{*}$ $=10^{-4}$.

We prepared a supercritical state at $T=1.1 T_{c}$ and $\langle n\rangle=n_{c}$ in equilibrium, where $\langle n\rangle=\int d \boldsymbol{r} n / V$ is the average density. We then lowered the temperature of all the boundary walls from $1.1 T_{c}$ to $0.9 T_{c}$ at $t=0$. Subsequently, a liquid wetting layer thickened and acted as a piston adiabatically expanding the interior. Here the interior was in a gaseous state with $n<n_{c}$ (see Fig. 2). In Fig. 1, we show snapshots of the density $n$, where instability and coarsening proceeded from the boundary into the interior on a slow time scale. It is remarkable that the interior can be adiabatically quenched on a rapid time scale. In Fig. 2, we display $T, n, s$, and the inverse isothermal compressibility,

$$
\frac{1}{K_{T}}=n\left(\frac{\partial p}{\partial n}\right)_{T}=\frac{k_{B} n}{\left(1-v_{0} n\right)^{2}}\left(T-T_{s}\right),
$$

at the cell center $x=y=L / 2$ in the early stage when the interior was still homogeneous. It demonstrates that the adiabatic changes were caused by sounds emitted from the boundary walls. In the present case, the isothermal compressibility be- 


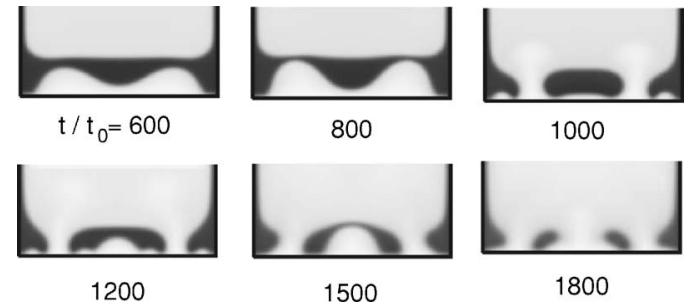

FIG. 10. Evaporation of a liquid layer at $g^{*}=10^{-4}$ after increasing the bottom temperature from $0.91 T_{c}$ to $1.1 T_{c}$, where the initial state is given in Fig. 9.

came negative or brought below the spinodal $T<T_{s}(n)$ in the early stage. The acoustic time scale $L / c$ is of order $\sigma^{1 / 2} t_{0} L / \ell \sim 10 t_{0}$ from Eqs. (3.8) and (3.9), which agrees with the oscillation period in Fig. 2.

We make some comments. (i) Though the interior was transiently close to the critical point in the adiabatic quenching in Fig. 2, we neglected the critical fluctuations and the critical anomaly of the transport coefficients. (ii) Though not shown here, temperature variations are significant among domains in the presence of latent heat, while previous simulations of spinodal decomposition have been performed in the isothermal condition $[6,27,39]$. (iii) In our case, a wetting layer acts as a piston because of its thickening in deep quenching. In near-critical fluids a thermal diffusion layer can well play the role of a piston in supercritical states. If the bulk region is in a liquid state below $T_{c}$, a cooled thermal diffusion layer can even cause phase separation in the interior [54]. By cooling the boundary wall at fixed volume, Moldover et al. [53] could realize homogeneous nucleation in the bulk at liquid densities but could not do so at gas densities, where the latter result was ascribed to the preexistence of a liquid wetting layer. The growth process of a liquid wetting layer upon cooling itself has not yet been examined in the literature.

\section{Piston effect with a bubble in liquid at $g=0$}

Let us consider the piston effect in the presence of a bubble in liquid. When $T_{b}$ is changed by $\delta T_{b}$, sound waves emitted from the boundary cause a pressure change $\delta p$. After many traversals of the sounds in the cell, $\delta p$ soon reaches of the following order [52]:

$$
\delta p \sim A_{s \ell}^{-1} \delta T_{b},
$$

where $A_{s \ell}$ is the liquid value of the adiabatic coefficient $A_{s}$ $=(\partial T / \partial p)_{s}$. From Eqs. (2.3) and (2.4) the van der Waals theory gives

$$
A_{s}=\left(\frac{\partial T}{\partial p}\right)_{s}=\frac{1 / n-v_{0}}{k_{B}\left[(1+d / 2)-d T_{s} / 2 T\right]} .
$$

If $\delta T_{b} \sim 0.1 T_{c}$ and $T / T_{c} \sim 0.9$, we find $\delta p \sim 0.1 p_{c}$, which is 10 times larger than the Laplace pressure difference $\gamma / R$ for $R \sim 50 \ell$ [46]. Here, due to the different values of $A_{s}$ in the two phases, there arises a considerable temperature difference in the two regions,

$$
(\Delta T)_{g \ell}=\left(A_{s g}-A_{s \ell}\right) \delta p,
$$

in the early stage. For example, $A_{s} k_{B} / v_{0}$ is equal to 7.1 for gas and 0.65 for liquid at $T=0.875 T_{c}$ from Eq. (3.14) in two dimensions. On the other hand, near the critical point, $A_{s g}$ $-A_{s \ell}$ tends to zero as $2 \gamma_{s}^{-1 / 2}(\partial T / \partial p)_{V}$, where $\gamma_{s}$ is the specific heat ratio [6]. Thus, the bubble temperature becomes higher (lower) than in the surrounding liquid for positive (negative) $\delta T_{b}$. For the case of a liquid droplet, as will be shown in Fig. 6 , the droplet temperature becomes lower (higher) than in the surrounding gas for positive (negative) $\delta T_{b}$.

In Figs. 3 and 4, we prepared a bubble with radius $R$ $=50 \ell=L / 4$ in equilibrium at $T=0.875 T_{c}$ under zero gravity. We then changed $T_{b}$ to $0.895 T_{c}$ for $t>0$ with $T_{t}$ held unchanged. In the upper plate of Fig. 3 , the bubble center is more heated than in the liquid at $y=L / 5$ and $4 L / 5$ in the early stage in accord with Eq. (3.15). We notice that the oscillation persists within the bubble for a longer time than in the liquid region. The lower panel of Fig. 3 shows the velocity field induced by the sounds at $t=55 t_{0}$, which is larger within the bubble than outside it. The typical velocity within the bubble is a few times larger than $\sqrt{\left\langle\boldsymbol{v}^{2}\right\rangle}$ $=0.011 \mathrm{\ell} / \mathrm{t}_{0}$ at this time. It indicates the presence of appreciable mass flux through the interface and suggests existence of collective modes with evaporation and condensation localized near a bubble. In Fig. 4, we show $\left(T-T_{t}\right) /\left(T_{b}-T_{t}\right)$, $p / p_{c}$, and $v_{0} n$ vs $y / L$ at $t=200 t_{0}$ and $x=L / 2$. Here, the oscillation is mostly damped, while the temperature inside the bubble is not yet flat. We also notice that the thermal diffusion layer is thickened up to the bubble, since the distance between the bottom and the bubble is narrow. It would take an extremely long time for macroscopic separation. While the temperature is continuous, the pressure is slightly higher inside the bubble than outside it by $\gamma / R \sim 0.01 p_{c}$ and exhibits a singularity in the interface region. The latter behavior can be seen in the equilibrium relation (2.10), from which the integral of $p$ in the interface region in the normal direction is equal to $-3 \gamma / 2$ for constant $M$.

\section{Two-phase states in heat flow at $g=0$}

In our previous work $[15,16]$, we examined the temperature and velocity field around a droplet in heat flow. We summarize the main results there. Under zero gravity, we showed numerically and analytically that a gas (liquid) droplet slowly moves to the warmer (cooler) boundary and that the temperature becomes nearly homogeneous within the droplet. Let a gas droplet (bubble) with radius $R$ be placed in a heat flux $Q=-\lambda_{\ell} T_{\infty}^{\prime}$, where $\lambda_{\ell}$ is the liquid thermal conductivity and $T_{\infty}^{\prime}$ is the temperature gradient far from the droplet. A convective velocity of order

$$
v_{c}=Q / n_{g} T \Delta s=-\lambda_{\ell} T_{\infty}^{\prime} / n_{g} T \Delta s
$$

is induced within the droplet, where $\Delta s=s_{g}-s_{\ell}$ is the entropy difference per particle between the two phases. The velocity $v_{c}$ characterizes latent heat flow and is independent of the bubble radius. The Reynolds number $\operatorname{Re}=v_{c} \rho_{g} R / \eta_{g}$ is less than unity for $R<\eta_{g} T \Delta s / m Q$, where $\rho_{g}=m n_{g}$ and $\eta_{g}$ are the mass density and the shear viscosity, respectively, in the gas phase. 
In our simulation $\mathrm{Re}$ is smaller than unity. In Figs. 5-8, we examine heat-conducting two-phase states at zero gravity, where the top temperature at $y=L$ is fixed at $T_{t}=0.875 T_{c}$ and the bottom temperature $T_{b}$ at $y=0$ is changed to a higher value. In Fig. 5, we initially prepared a bubble with radius $R=50 \ell$ in equilibrium at $T=0.875 T_{c}$ at the cell center. We then raised $T_{b}$ to a high value, $0.945 T_{c}$, for $t>0$ to induce droplet motion toward the bottom. The time scale of migration was about $10^{4} t_{0}$. Eventually, the droplet partially wetted the bottom plate with an apparent contact angle. However, Fig. 5 shows the presence of a thin gas layer sandwiched between them. The typical velocity in Fig. 5 is given by $\sqrt{\left\langle\boldsymbol{v}^{2}\right\rangle}=0.029 \ell / t_{0}$, which is of order $v_{c}$ in Eq. (3.16). We can see that $T$ is flat inside the bubble and continuous across the interface. The generalized chemical potential $\hat{\mu}$ in Eq. (2.18) exhibits the same behavior (not shown here). The pressure is nearly constant outside the bubble at $p \cong 0.76 p_{c}$, while it is slightly higher inside by $\gamma / R \sim 0.01 p_{c}$ [46]. With further increasing $T_{b}$, the apparent contact angle decreases and the bottom wall is eventually wetted completely by gas. See such an example of complete wetting at $T_{b}=0.97 T_{c}$ in Ref. [15]. As a related experiment, Garrabos et al. observed that gas spreads on a heated wall initially wetted by liquid and exhibits an apparent contact angle [10].

Next, we initially prepared a liquid droplet $\mathrm{i}$ gas with $R$ $=50 \ell$ at the cell center at $T=0.875 T_{c}$ and then raised $T_{b}$ to $0.91 T_{c}$ for $t>0$. In Fig. 6, we show $T, \boldsymbol{v}$, and $n$ around a liquid droplet suspended in gas at $t=4565 t_{0}$. Here the velocity field is nearly steady and, as in the lower panel of Fig. 3, it is much smaller in the liquid than that in the gas due to the viscosity difference from Eq. (3.3). The average velocity amplitude is given by $\sqrt{\left\langle\boldsymbol{v}^{2}\right\rangle}=0.015 \ell / t_{0}$ at this time. The temperature inside the droplet is nearly homogeneous and is lowest in the cell, while the pressure inside it is again only slightly higher than outside it by $\gamma / R \sim 0.01 p_{c}$. Hence, condensation is taking place at the interface and the temperature inside it is slowly increasing in time as $0.849 T_{c}, 0.851 T_{c}$, and $0.855 T_{c}$ at $t / t_{0}=4565,7770$, and 15820 , respectively, on the time scale of thermal diffusion $\left(\sim R^{2} / D \sim t_{0} R^{2} / \ell^{2}\right)$. Note that the initial temperature lowering inside the liquid droplet can be explained by the adiabatic relation (3.15) (see the arguments around it). In Fig. 7, we show three snapshots of the density at later times in the same run as in Fig. 6. The droplet migration to the cooler wall is very slow, but after its encounter with it the liquid spreads over it rather rapidly under the wetting condition (3.2). In this process the warmer boundary remains dried.

\section{E. Heat conduction in steady two-phase states}

Even when the liquid volume fraction is small, efficient heat transport is realized in the presence of evaporation on the warmer boundary and/or condensation on the cooler boundary. This is the mechanism of heat pipes widely used in refrigerators and air conditioners. In such processes, the warmer boundary drys out if evaporation proceeds without supply of liquid. Here we demonstrate that steady heat transport with evaporation and condensation can be achieved in a closed cell in the presence of a liquid wetting layer and/or a gravity.
In our situation, we define the effective thermal conductivity through the cell by

$$
\lambda_{\text {eff }}=\int_{0}^{L} d x Q_{b}(x) /\left(T_{b}-T_{t}\right) .
$$

As the heat flux, we choose its value at the bottom $y=0$,

$$
Q_{b}(x)=-(\lambda \partial T / \partial y)_{y=0},
$$

where $\boldsymbol{v}$ vanishes. It is the heat flux from the bottom boundary wall to the fluid. In convection, a normalized effective thermal conductivity, called the Nusselt number, has been used to represent the efficiency of heat transport. We define it by

$$
N u=\lambda_{\text {eff }} / \lambda_{\ell} .
$$

We take $\lambda_{\ell}$ as the thermal conductivity at $n=1.7 n_{c}$, which is the liquid density in two-phase coexistence at $T=0.875 T_{c}$ [see the sentences below Eq. (3.4)]. In one-phase states without convection, we have $N u=n / 1.7 n_{c}$ under Eq. (3.4). Therefore, $N u$ is small for gas without convection.

In Fig. 8, we show $T, \boldsymbol{v}$, and $n$ in a steady heat conducting state with $T_{b}=0.89 T_{c}$ and $T_{t}=0.875 T_{c}$ at zero gravity. A characteristic feature in this case is that a liquid layer covers all the boundary walls. The average density $\langle n\rangle=0.22 v_{0}^{-1}$ is relatively small. The typical velocity is given by $\sqrt{\left\langle\boldsymbol{v}^{2}\right\rangle}$ $=0.0080 \ell / t_{0}$. Remarkably, the temperature profile is nearly flat in the majority gas region, due to latent heat convection through the cell. As a result, $N u=5.0$ is realized, despite the fact that the liquid region is only near the boundary walls.

Under gravity, the warmer boundary does not dry out even for large heat flux. In Fig. 9, we realized a steady state with $\langle n\rangle=0.206 v_{0}^{-1}$ and $T_{b}=0.91 T_{c}$ under gravity at $g^{*}$ $=10^{-4}$, where $\sqrt{\left\langle\boldsymbol{v}^{2}\right\rangle}=0.0119 \ell / t_{0}$ and $N u=2.0$. Again a liquid layer covers all the boundary walls, while the bottom part is thicker due to the gravity. These features are very different from those in the right panel of Fig. 7 without gravity. In Fig. 9 , the temperature gradient is large everywhere on the side walls and widely in the region $y / \ell \leqq 0.25$ near the bottom, while the liquid-gas interface is at $y / \ell \sim 0.1$ apart from the side walls. Due to these features we obtain the smaller value of $N u$ than for the case in Fig. 8. In Fig. 10, we raised $T_{b} / T_{c}$ from 0.91 to 1.1 starting with the steady state in Fig. 9. We recognize that this is the case of strong heating, where bubbles emerge from the bottom to break the liquid into smaller pieces. As the gas flow from the bottom is warmer, condensation occurs on the side and top boundary walls, leading to growth of the liquid wetting layer there.

\section{F. Boiling in gravity}

Boiling phenomena are very complex and still remain virtually unexplored in physics [6,12-14]. Usually, the typical bubble size is macroscopic and the associated Reynolds number is large. In the present case, we realized boiling under gravity at $g^{*}=10^{-4}$ even for our small system size, where bubbles from the heated bottom wall were small but could rise upward with small Reynolds numbers.

In Figs. 11 and 12, we set $\langle n\rangle=0.34 v_{0}^{-1}$ and held $T_{t}$ at $0.775 T_{c}$. Starting with an equilibrium two-phase state at $t$ 


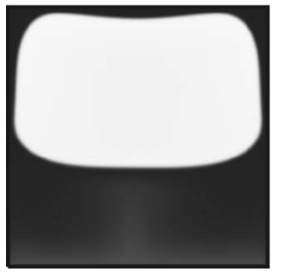

$t / t_{0}=10000$

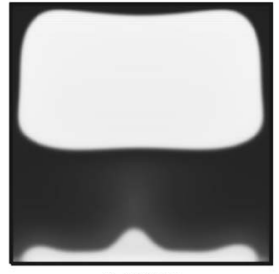

34600

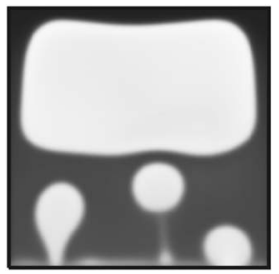

66300

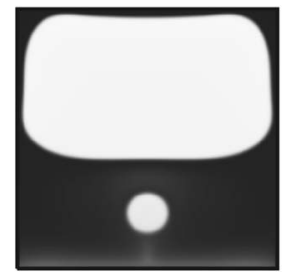

27700

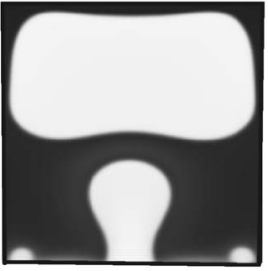

35100

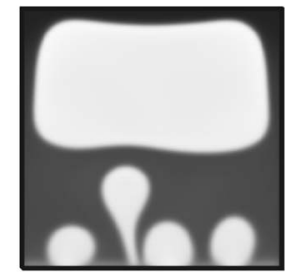

71000

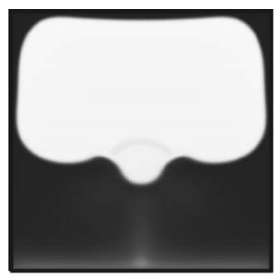

28010

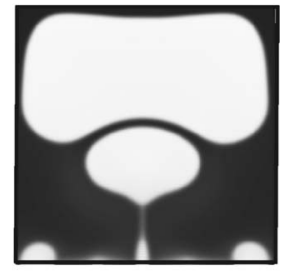

35230

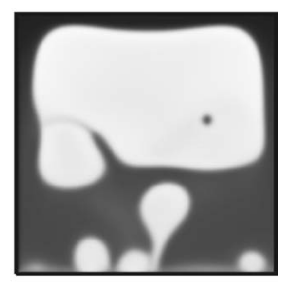

74100
FIG. 11. Changeovers of boiling with increasing $T_{b}$ at $T_{t}$ $=0.775 T_{c}$ and $g^{*}=10^{-4}$. Upper plates: nonboiling state at $T_{b}$ $=0.91 T_{c}$ (left), and weakly boiling state with a single bubble at $T_{b}$ $=0.945 T_{c}$ (middle and right). Middle plates: transition from weak to strong boiling after a change of $T_{b}$ from $0.945 T_{c}$ to $1.0 T_{c}$ at $t$ $=34245 t_{0}$. Lower plates: dynamical steady state with continuous bubble formation and rising.

$=0$, we reached a steady nonboiling state at $T_{b}=0.915 T_{c}$, where $\sqrt{\left\langle\boldsymbol{v}^{2}\right\rangle}=0.023 \ell / t_{0}$ and $N u=3.2$ owing to latent heat convection in the gas region. See the left snapshot at $t$ $=10^{4} t_{0}$ in the upper panels of Fig. 11. We then raised $T_{b}$ to $0.945 T_{c}$ to produce a weakly boiling state, where a single bubble periodically appeared with a period about $10^{3} t_{0}$. At $t=27700 t_{0}$ a bubble is at the center of the liquid region, while at $t=28010 t_{0}$ it is being absorbed into the gas region. Here $\sqrt{\left\langle\boldsymbol{v}^{2}\right\rangle}=0.033 \ell / t_{0}$ and $N u=4.5$ at $t=27700 t_{0}$. Finally, at $t=t_{\mathrm{b}}=34245 t_{0}$, we again raised $T_{b}$ to $1.0 T_{c}$. The middle panels of Fig. 11 display transition from weak to strong boiling. The bottom plates of Fig. 11 are those in fully developed boiling, which was reached for $t-t_{b} \geqslant 5000 t_{0}$. In Fig. 12, we show $T, n$, and $\boldsymbol{v}$ in fully developed boiling at $t=63047 t_{0}$, where $\sqrt{\left\langle\boldsymbol{v}^{2}\right\rangle}=0.055 \ell / t_{0}$ and $N u=9.2$. We can see steep temperature gradients near the boundary walls and large velocity deviations around the rising bubbles. In Fig. 13, we show that the heat flux $Q_{b}(x)$ on the bottom in Eq. (3.18) is large where rising bubbles are being produced. The upper panel at $t=34600 t_{0}$ is at the inception of strong boiling, exhibiting a sharp maximum where a bubble is being detached. The lower panel of Fig. 13 at $t=63047 t_{0}$ is taken in the fully developed boiling state, exhibiting multiple peaks.

In our boiling simulation, the bubble sizes are of order $R \sim 20 \ell$ and the Reynolds number $\operatorname{Re}=\sqrt{\left\langle\boldsymbol{v}^{2}\right\rangle} R / \nu_{0}$ on the droplet scale is of order 0.1 in the lower panels in Fig. 11 and in Fig. 12. Thanks to the high efficiency of latent heat transport, the temperature gradient is mostly localized near the boundary walls. Generally in gravity, there should remain a small temperature gradient in the bulk region in boiling and in stirring. It is given by the so-called adiabatic temperature gradient $[6,14]$,

$$
\left(\frac{d T}{d z}\right)_{\text {bulk }}=-\rho g\left(\frac{\partial T}{\partial p}\right)_{s},
$$

which is the minimum for the onset of convection for compressible fluids [47] and is equal to $-0.27 \mathrm{mK} / \mathrm{cm}$ for $\mathrm{CO}_{2}$ near the critical point. In our case, the right-hand side of Eq. (3.20) is of order $-g^{*} T_{c} / \ell$ and is consistent with the average gradient seen in the upper panel of Fig. 12. In usual Rayleigh-Bénard convection in one-phase fluids, the average temperature gradient in the bulk region can be suppressed only in turbulence by rapid convective motion of thermal plumes, where the Reynolds number exceeds unity even on the scale of the plumes [56].

\section{G. Wetting dynamics}

Wetting dynamics has been studied mostly for involatile droplets [7], but it has not yet been well understood for volatile droplets or in the presence of evaporation and condensation [8-10]. The previous approaches in the latter case were by solving the hydrodynamic equations with phenomenological surface boundary conditions [8] and by performing molecular dynamics simulations of nanometer-size liquid droplets [9]. Here we present its preliminary numerical study in our scheme.

At $t=0$, we placed a semi-circular liquid droplet with $R$ $=40 \ell$ on the bottom, where the interface was on the curve $(x-L / 2)^{2}+y^{2}=R^{2}$. The initial temperature was $0.875 T_{c}$ throughout the cell. In the upper panels of Fig. 14, where both $T_{b}$ and $T_{t}$ were held unchanged, spreading of the liquid region occurred over the walls on a time scale of $10^{3} t_{0}$. In the middle panels of Fig. 14, we set $T_{b}=0.915 T_{c}$ for $t>0$ to induce slow evaporation of the liquid region. We can see only a small amount of liquid left on the bottom at $t$ $=4000 t_{0}$ (right). In the bottom panels of Fig. 14, where $T_{b}$ $=0.975 T_{c}$ for $t>0$, a gas droplet appeared within the liquid region and, as it grew, the liquid region itself was finally detached from the boundary (right). In these processes, a considerable amount of liquid was evaporating even for the first case of $T_{b}=T_{t}=0.875 T_{c}$ and the latent heat needed for evaporation was supplied from the equitemperature boundary wall. In all these examples, the liquid region was itself cooled even below the top temperature $T_{t}=0.875 T_{c}$ by an amount typically of order $0.05 T_{c}$. In Fig. 15, we show the velocity $\boldsymbol{v}$ (upper panel) and the heat flux $Q_{b}(x)$ (lower panel) at $t=500 t_{0}$ obtained from the run giving the series (a) in Fig. 14. We can see a circulating flow in the gas and large heat absorption at the contact points. The peak height of $Q_{b}(x)$ gradually decreased with spreading. These stem from evaporation at the contact points and condensation on the top of the droplet. The flow pattern here is very different from that for involatile droplets [7]. 

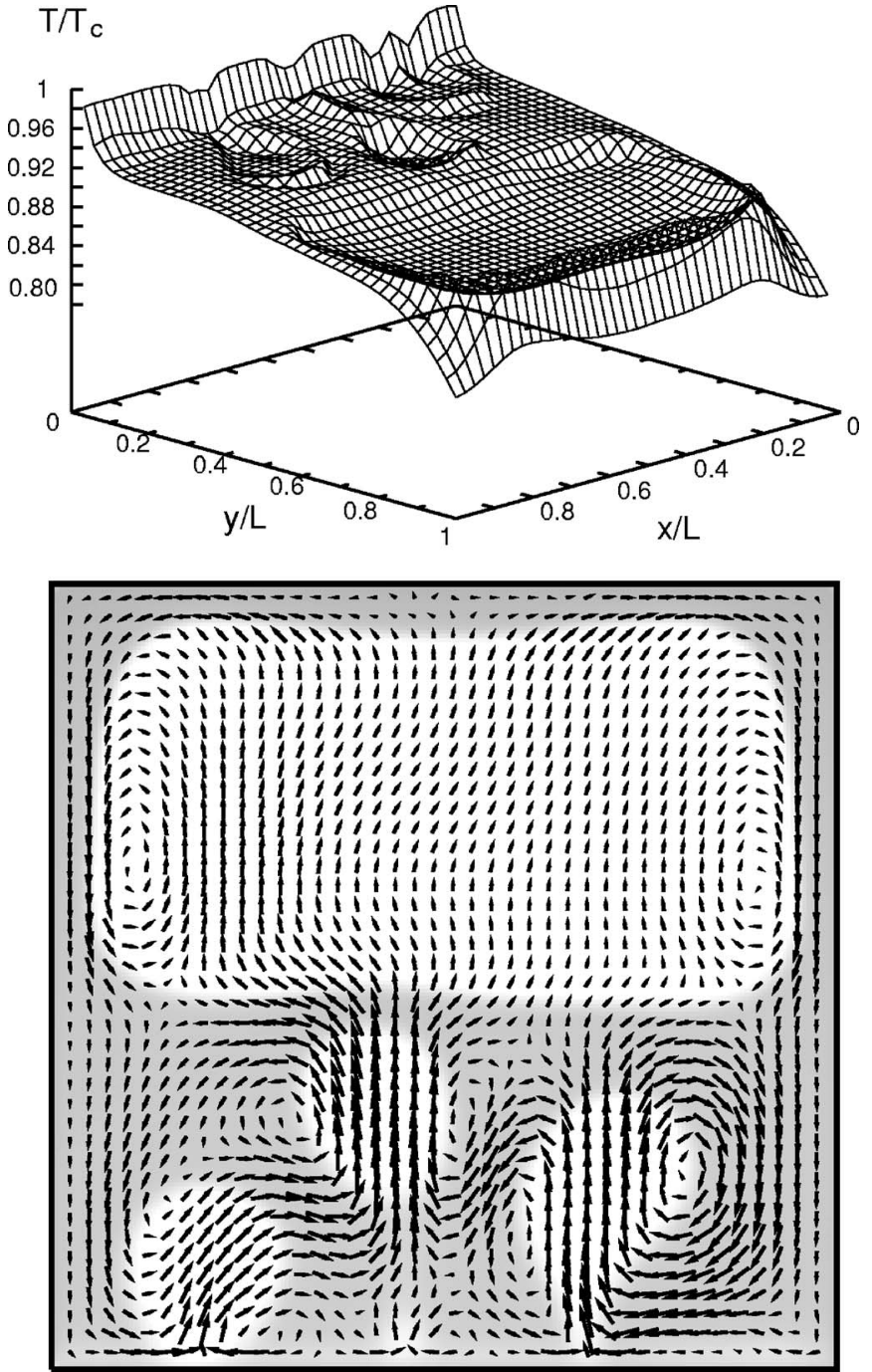

FIG. 12. Temperature (upper plate) and velocity field (lower plate) in a fully developed boiling state at $T_{b}=T_{c}$ and $T_{t}=0.775 T_{c}$ under $g^{*}=10^{-4}$ at $t=63047 t_{0}$.

We mention two theoretical papers in accord with our results. Koplik et al. found a large temperature decrease of a nanometer-scale liquid droplet on a heated wall using molecular dynamics simulation [9]. Nikolayev et al. solved the heat conduction equation around a contact point of a bubble in two dimensions to find a divergence of $Q_{b}(x)$ there on an equitemperature heated wall [11].

\section{SUMMARY}

We have developed a general scheme of the dynamic van der Waals theory. Our hydrodynamic equations including the gradient stress can readily be integrated numerically. We may then gain intuitive ideas of complicated but fundamental hydrodynamic processes in two-phase states. In particular, emphasis has been put on adiabatic changes and latent heat transport in two-phase states, which have rarely been discussed in the literature. Though in two dimensions and for small system size $(200 \times 200)$, we have presented a number
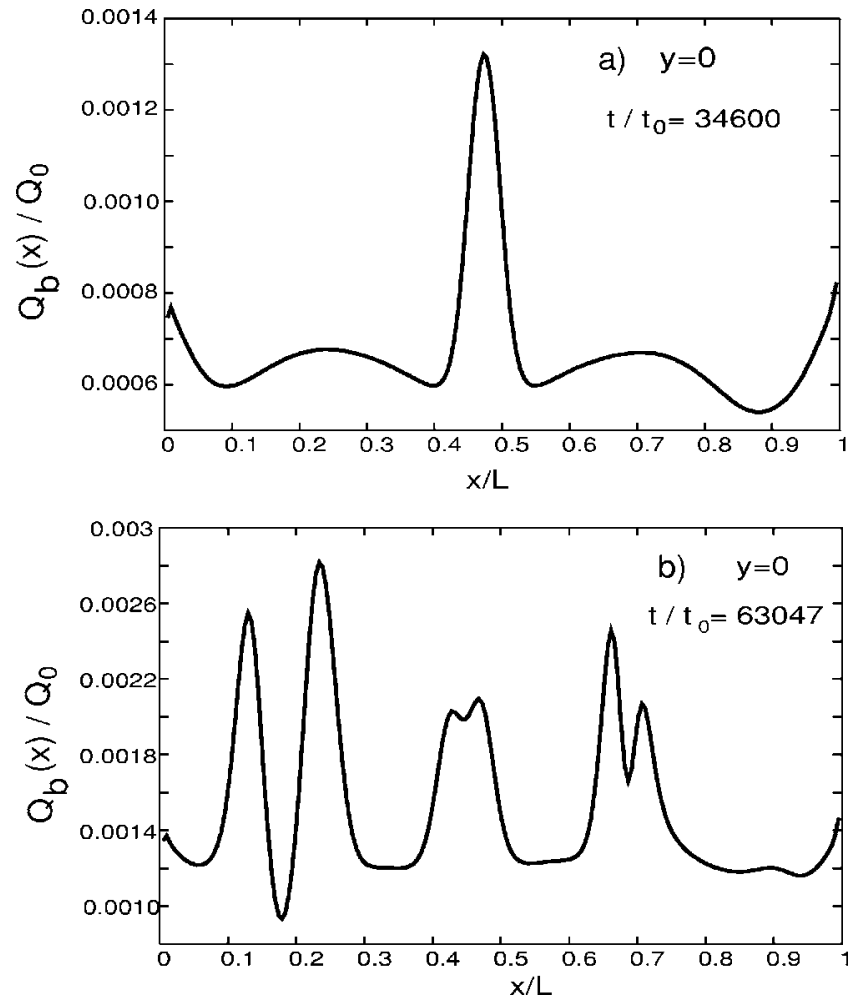

FIG. 13. Normalized heat flux $Q_{b}(x) / Q_{0}$ at the bottom in boiling at $t=34600 t_{0}$ in (a) and $t=63047 t_{0}$ in (b), corresponding to the first panel in Fig. 11 for (a) and to Fig. 12 for (b). Here $Q_{b}(x)$ is defined by Eq. (3.18) and $Q_{0}=\epsilon \ell / v_{0} t_{0}$.

of simulation results to demonstrate the usefulness of our scheme. Examples treated are (i) adiabatically induced spinodal decomposition, (ii) piston effect with a bubble in liquid, (iii) temperature and velocity profiles around a gas and liquid droplet in heat flow, (iv) efficient latent heat transport at small liquid densities, (v) boiling in gravity with continuous bubble formation and rising, and (vi) spreading and evaporation of a liquid region on a heated boundary wall.

We make some comments below.

(i) Our numerical analysis in this work is very fragmentary. We should investigate each problem in more detail over wide ranges of the parameters. In particular, we have used the special boundary condition (3.2), for which liquid is strongly attracted to the boundary walls. We should perform more simulations for various $a_{s}$ and $b_{s}$ in Eq. (2.33).

(ii) The mesh size of our simulation cell $\ell$ is on the order of the interface thickness away from the critical point. If $\ell$ is a microscopic length in our numerical analysis, we have treated hydrodynamics on small scales $(\sim$ several 10 nanometers), as in the molecular dynamics simulation [9]. We have not examined crossovers taking place with increasing the spatial scales, but general features of adiabatic changes and latent heat transport revealed in this work should be independent of the spatial scales.

(iii) In integrating our hydrodynamic equations, we do not assume phenomenological boundary conditions at the interface, while they are needed in the hydrodynamic calculation in the thin interface limit $[8,11,50]$. To clarify the relationship between these two approaches, we should examine the 
a)
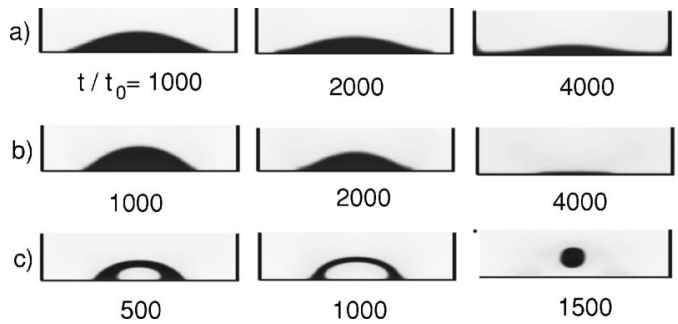

FIG. 14. Spreading and evaporation of a liquid region on the bottom with $T_{b} / T_{c}=0.875$ in (a) (top), 0.915 in (b) (middle), and 0.975 in (c) (bottom), with the initial temperature being at $0.875 T_{c}$.

thin interface limit of our model [26-28]. Moreover, in the phase field model of crystal growth [31-33], the interface motion can be insensitive to the interface thickness $\ell$ under some conditions among the parameters in the model. Also in our fluid problems, we should clarify such conditions.

(iv) In our numerical analysis, we evidently see smooth variations of the temperature $T$ and the generalized chemical potential $\hat{\mu}$ in Eq. (2.18) across the interface (not shown for $\hat{\mu})$. In our hydrodynamic theory [16], we assumed the continuity of these quantities together with the stress balance and the mass and energy conservation at the interface. In the literature $[8,29]$, however, discontinuities in the temperature and the chemical potential have been supposed to arise across the interface between liquid and dilute gas (which originate from the long mean free path in the gas phase).

(v) Since the Reynolds number is small in this work, we should examine the high-Reynolds-number regime by enlarging the droplet size $R$ in a much larger system [see the discussion below Eq. (3.16)].

(vi) Figure 3 indicates complex nonspherical dynamic response of bubbles to sound waves in the presence of evaporation and condensation. This aspect seems to have not yet been investigated, while large sound attenuation of liquid containing bubbles has long been discussed [6].

(vii) We should study hydrodynamics near the critical point, where the singularities of the thermodynamic and dynamical properties largely influence the dynamics $[6,10,12,14,54]$. It is well-known that the piston effect gives rise to critical speeding-up of thermal equilibration [52], while the thermal diffusion is slowed down. In near-critical one-phase convection, significant are overall pressure and temperature fluctuations propagating as sounds on birth and arrival of thermal plumes at the boundary walls [56]. In nearcritical two-phase states, such adiabatic fluctuations could be stronger in boiling and phase separation.

(viii) We are also interested in droplet motion and twophase hydrodynamics in binary fluid mixtures. Marek and Straub examined the effect of a small amount of noncondensable gas doped in the gas phase [43], which drastically alters thermocapillary convection. Pooley et al. [20] took account of latent heat transport in binary mixtures to calculate the Nusselt number in two-phase convection.

(ix) In the Landau and Lifshitz book [47], the linearized hydrodynamic equations are treated as Langevin equations, where newly added are random stress and random heat flux related to the transport coefficients via the fluctuationdissipation relations [6]. They describe the thermal fluctua-
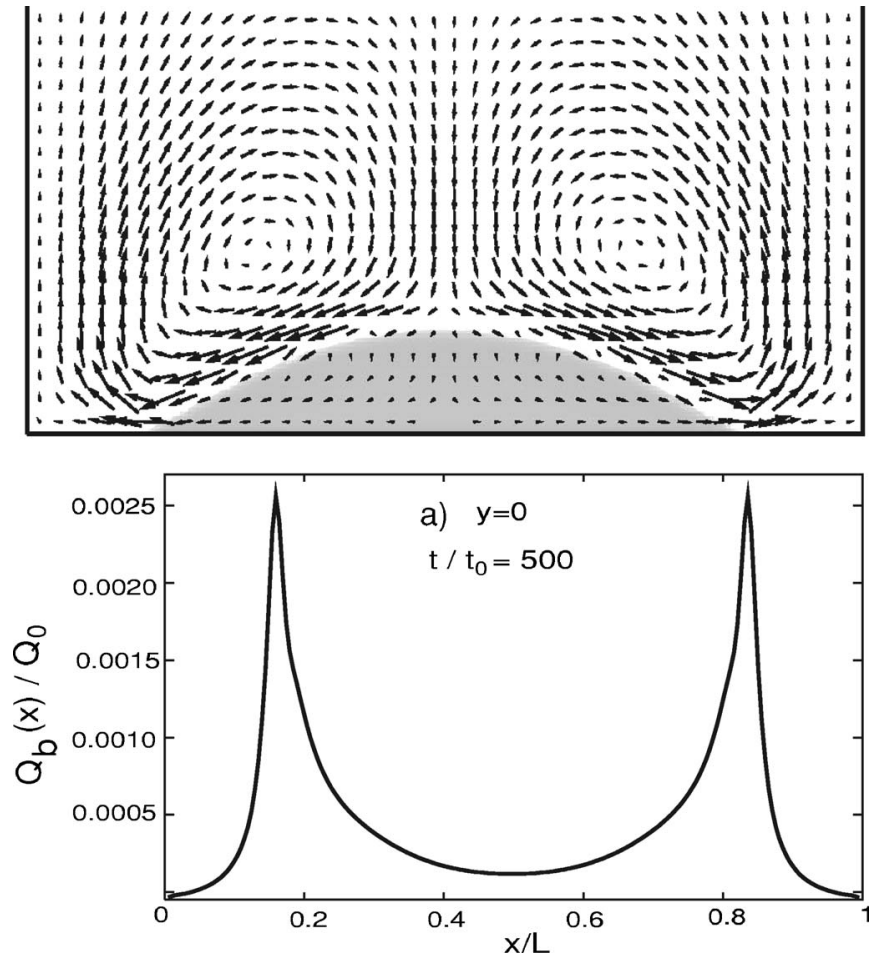

FIG. 15. Velocity $\boldsymbol{v}$ (upper panel) and normalized heat flux $Q_{b}(x) / Q_{0}$ at the bottom (lower panel) at $t=500 t_{0}$ with $T_{b} / T_{c}$ $=0.875$ obtained from the run giving the series (a) in Fig. 14. Here $Q_{0}=\epsilon \ell / v_{0} t_{0}$. Absorption is maximum at the contact points, where evaporation is taking place.

tions of the hydrodynamic variables. The random source terms in the dynamic equations are particularly important near the critical point $[5,6]$. We should clarify how our hydrodynamic equations with the gradient stress can be treated as Langevin equations.

(x) We mention the lattice-Boltzmann method devised to treat two-phase flow and phase separation $[20,57,58]$. In particular, Palmer and Rector included thermal effects to calculate a nonequilibrium temperature profile around a gas-liquid interface [58].

\section{ACKNOWLEDGMENTS}

This work was supported by Grants in Aid for Scientific Research and for the 21st Century COE project (Center for Diversity and Universality in Physics) from the Ministry of Education, Culture, Sports, Science and Technology of Japan. The author would like to thank D. Beysens, V. S. Nikolayev, and D. Bedeaux for valuable discussions. The author also thanks T. Hamanaka for pointing out an error in the simulation program used in Ref. [15].

\section{APPENDIX A: DERIVATION OF THE REVERSIBLE STRESS}

We derive the reversible stress tensor $\stackrel{\leftrightarrow}{\Pi}$ following the method in Appendix 6A of Ref. [6]. Adopting the Lagrange picture of fluid motion, we suppose a small fluid element 
with volume $V$ at position $\boldsymbol{r}$ and at time $t$. Due to the velocity field the element is displaced to a new position, $\boldsymbol{r}^{\prime}=\boldsymbol{r}+\boldsymbol{u}$ with $\boldsymbol{u}=\boldsymbol{v} \delta t$, after a small time interval $\delta t$. Hereafter, we neglect the dissipation $(\eta=\zeta=\lambda=0)$. The density $n$ and the volume of the fluid element $V$ are changed by

$$
\delta n=-n \boldsymbol{\nabla} \cdot \boldsymbol{u}, \quad \delta V=V \boldsymbol{\nabla} \cdot \boldsymbol{u} .
$$

The change of the internal energy in the volume element is written as $\delta \mathcal{E}=\int d \boldsymbol{r}^{\prime} \hat{e}^{\prime}-\int d \boldsymbol{r} \hat{e}=V \delta \hat{e}$, where $d \boldsymbol{r}^{\prime}=d \boldsymbol{r}(1+\boldsymbol{\nabla} \cdot \boldsymbol{u})$. From Eq. (2.39) we have

$$
\delta \hat{e}=-(\hat{e} \stackrel{\leftrightarrow}{I}+\stackrel{\leftrightarrow}{\Pi}): \nabla u,
$$

where $\overleftrightarrow{I}=\left\{\delta_{i j}\right\}$ is the unit tensor. We shift the coordinates as $\boldsymbol{r}^{\prime}=\boldsymbol{r}+\boldsymbol{u}$; then, the space derivatives are changed as

$$
\frac{\partial}{\partial x_{i}^{\prime}}=\frac{\partial}{\partial x_{i}}-\sum_{j} \frac{\partial u_{j}}{\partial x_{i}} \frac{\partial}{\partial x_{j}} .
$$

As a result, $|\nabla n|^{2} / 2$ is changed by $\Sigma_{i}\left(\nabla_{i} n\right)\left(\nabla_{i} \delta n\right)-\Sigma_{i j}\left(\nabla_{i} n\right)$ $\times\left(\nabla_{j} n\right)\left(\nabla_{i} u_{j}\right)=\boldsymbol{\nabla} n \cdot \nabla \delta n-\nabla n \nabla n: \nabla \boldsymbol{u}$. We then calculate the entropy change in the volume element $\delta \mathcal{S}=\int d \boldsymbol{r}^{\prime} \hat{s}^{\prime}-\int d \boldsymbol{r} \hat{s}$ as

$$
\frac{T}{V} \delta \mathcal{S}=[(T \hat{S}-\hat{e}+n \hat{\mu}) \stackrel{\leftrightarrow}{I}-\stackrel{\leftrightarrow}{\Pi}+M \nabla n \nabla n]: \nabla \boldsymbol{u} .
$$

Without dissipation we should require $\delta \mathcal{S}=0$ to obtain the reversible stress $\stackrel{\leftrightarrow}{\Pi}$ in Eqs. (2.47) and (2.48).

\section{APPENDIX B: SCALED HYDRODYNAMIC EQUATIONS}

We rewrite our hydrodynamic equations into dimensionless forms in two dimensions using Eqs. (3.3) and (3.4). The scaled space and time, $\ell^{-1} \boldsymbol{r}$ and $t_{0}^{-1} t$, will be simply written as $\boldsymbol{r}$ and $t$, respectively. Here we introduce the scaled density, temperature, and velocity,

$$
\phi=v_{0} n, \quad \Theta=\frac{k_{B}}{\epsilon} T, \quad V=\frac{t_{0}}{\ell} \boldsymbol{v} .
$$

The continuity equation (2.35) is written as $\partial \phi / \partial t=$ $-\boldsymbol{\nabla} \cdot(\phi \boldsymbol{V})$. The momentum equation (2.36) reads

$$
\sigma\left[\frac{\partial}{\partial t} \phi \boldsymbol{V}+\boldsymbol{\nabla} \cdot(\phi \boldsymbol{V} \boldsymbol{V})\right]=-\boldsymbol{\nabla} \cdot \overleftrightarrow{M}-g^{*} \phi \boldsymbol{e}_{z}
$$

where $\sigma$ and $g^{*}$ are the constants defined by Eqs. (3.6) and (3.7). Including the viscous stress we define

$$
\begin{aligned}
M_{i j}= & {\left[\frac{\Theta \phi}{1-\phi}-\phi^{2}-2 \Theta \nabla^{2} \phi-\Theta(\nabla \phi)^{2}\right] \delta_{i j} } \\
& +2 \Theta\left(\nabla_{i} \phi\right)\left(\nabla_{j} \phi\right)-\sigma \phi\left(\nabla_{i} V_{j}+\nabla_{j} V_{i}\right) .
\end{aligned}
$$

We define a scaled total internal energy density by $E_{T}$ $\equiv e_{T} v_{0} / \epsilon$ to obtain

$$
E_{T}=\phi \Theta-\phi^{2}+\frac{\sigma}{2} \phi V^{2},
$$

which is governed by

$$
\frac{\partial}{\partial t} E_{T}+\boldsymbol{\nabla} \cdot\left(E_{T} \boldsymbol{v}+\overleftrightarrow{M} \cdot \boldsymbol{V}\right)=\boldsymbol{\nabla} \cdot \phi \boldsymbol{\nabla} T-g^{*} \phi V_{z} .
$$

The boundary condition (3.2) is rewritten as

$$
\boldsymbol{\nu} \cdot \boldsymbol{\nabla} \phi=(\phi-5 / 6) / 2 \sqrt{2} \text {. }
$$

Equations (2.35), (B2), and (B5) were integrated using the Euler scheme with the time mesh size $\Delta t=0.01$ [59].
[1] J. D. van der Waals, Verh.-K. Ned. Akad. Wet., Afd. Natuurkd., Eerste Reeks 1(8), 56 (1893); J. S. Rowlinson, [J. Stat. Phys. 20, 197 (1979).

[2] N. van Kampen, Phys. Rev. 135, A362 (1964).

[3] V. L. Ginzburg and L. D. Landau, Zh. Eksp. Teor. Fiz. 20, 1064 (1950).

[4] J. W. Cahn and J. E. Hilliard, J. Chem. Phys. 28, 258 (1958).

[5] P. C. Hohenberg and B. I. Halperin, Rev. Mod. Phys. 49, 435 (1977).

[6] A. Onuki, Phase Transition Dynamics (Cambridge University Press, Cambridge, 2002).

[7] P. G. de Gennes, Rev. Mod. Phys. 57, 827 (1985).

[8] D. M. Anderson and S. H. Davis, Phys. Fluids 7, 248 (1995); A. Oron, S. H. Davis, and S. G. Bankoff, Rev. Mod. Phys. 69, 931 (1997).

[9] J. X. Yang, J. Koplik, and J. R. Banavar, Phys. Rev. Lett. 67, 3539 (1991); J. Koplik, S. Pal, and J. R. Banavar, Phys. Rev. E 65, 021504 (2002).

[10] Y. Garrabos, C. Lecoutre-Chabot, J. Hegseth, V. S. Nikolayev, D. Beysens, and J.-P. Delville, Phys. Rev. E 64, 051602 (2001); J. Hegseth, A. Oprisan, Y. Garrabos, V. S. Nikolayev, C. Lecoutre-Chabot, and D. Beysens, ibid. 72, 031602 (2005).
[11] V. S. Nikolayev, D. A. Beysens, G.-L, Lagier, and J. Hegseth, Int. J. Heat Mass Transfer 44, 3499 (2001).

[12] V. S. Nikolayev and D. A. Beysens, Europhys. Lett. 47, 345 (1999).

[13] J. Straub, Int. J. Therm. Sci. 39, 490 (2000).

[14] A. Onuki, Physica A 314, 419 (2002).

[15] A. Onuki, Phys. Rev. Lett. 94, 054501 (2005). In this paper the gradient stress is written with the opposite sign. Also there is an error in the computer program such rotational motion of flow is incorrectly superposed.

[16] A. Onuki and K. Kanatani, Phys. Rev. E 72, 066304 (2005).

[17] S. Sakurai, A. Tschammer, W. Pesch, and G. Ahlers, Phys. Rev. E 60, 539 (1999).

[18] M. Assenheimer, B. Khaykovich, and V. Steinberg, Physica A 208, 373 (1994).

[19] T. Araki and H. Tanaka, Europhys. Lett. 65, 214 (2004).

[20] C. M. Pooley, O. Kuksenok, and A. C. Balazs, Phys. Rev. E 71, 030501(R) (2005).

[21] A. Onuki, J. Low Temp. Phys. 50, 433 (1983); 55, 309 (1984).

[22] R. V. Duncan, G. Ahlers, and V. Steinberg, Phys. Rev. Lett. 60, 1522 (1988); W. A. Moeur, P. K. Day, F.-C. Liu, S. T. P. Boyd, 
M. J. Adriaans and R. V. Duncan, ibid. 78, 2421 (1997).

[23] D. Korteweg, Arch. Neerl. Sci. Exactes Nat., Ser. II 6, 1 (1901)

[24] H. Gouin, in PhysicoChemical Hydrodynamics: Interfacial Phenomena, edited byM. G. Veralde, NATO Advanced Studies Institute, Series B: Physics (Plenum, New York, 1988), Vol. 174, p. 667; P. Casal and H. Gouin, C. R. Acad. Sci., Ser. II: Mec., Phys., Chim., Sci. Terre Univers 306(2), 99 (1988).

[25] D. D. Joseph, Eur. J. Mech. B/Fluids 9, 565 (1990); D. D. Joseph, A. Huang, and H. Hu, Physica D 97, 104 (1996).

[26] D. M. Anderson, G. B. McFadden, and A. A. Wheeler, Annu. Rev. Fluid Mech. 30, 139 (1998).

[27] D. Jasnow and J. Viñals, Phys. Fluids 8, 660 (1996); R. Chella and J. Viñals, Phys. Rev. E 53, 3832 (1996).

[28] D. Jamet, O. Lebaigue, N. Coutris, and J. M. Delhaye, J. Comput. Phys. 169, 624 (2001).

[29] D. Bedeaux, E. Johannessen, and A. Rosjorde, Physica A 330, 329 (2003); E. Johannessen and D. Bedeaux, ibid. 330, 354 (2003); 336, 252 (2004).

[30] A. Asai, K. Shinjo, and S. Miyashita, J. Phys. Soc. Jpn. 75, 024001 (2006).

[31] R. Kobayashi, Physica D 63, 410 (1993).

[32] G. Caginalp, Phys. Rev. A 39, 5887 (1989); A. Karma and W. J. Rappel, Phys. Rev. E 57, 4323 (1998).

[33] D. M. Anderson, G. B. McFadden, and A. A. Wheeler, Physica D 135, 175 (2000).

[34] M. Fixman, J. Chem. Phys. 47, 2808 (1967).

[35] L. P. Kadanoff and J. Swift, Phys. Rev. 166, 89 (1968).

[36] K. Kawasaki, Prog. Theor. Phys. 41, 1190 (1969).

[37] B. U. Felderhof, Physica (Amsterdam) 48, 541 (1970).

[38] R. Perl and R. A. Ferrell, Phys. Rev. A 6, 2358 (1972).

[39] T. Ohta and K. Kawasaki, Prog. Theor. Phys. 59, 362 (1978).

[40] A. Onuki, Phys. Rev. A 35, 5149 (1987).

[41] Pep Espaöl, J. Chem. Phys. 115, 5392 (2001).

[42] L. P. Pitaevskii, Zh. Eksp. Teor. Fiz. 40, 646 (1961) [Sov. Phys. JETP 13, 451 (1961)].

[43] R. Marek and J. Straub, Int. J. Heat Mass Transfer 44, 619 (2002).

[44] For diatomic molecules such as $\mathrm{O}_{2}$ the kinetic energy contribution to $e$ should be $5 n k_{B} T / 2$ due to the two rotational degrees of freedom. For polyatomic molecules such as $\mathrm{CO}_{2}$ it should be $3 n k_{B} T$.

[45] You-Xiang Zuo and E. H. Stenby, Fluid Phase Equilib. 132, 139 (1997); S. B. Kiselev and J. F. Ely, J. Chem. Phys. 119, 8645 (2003).
[46] H. Kitamura and A. Onuki, J. Chem. Phys. 123, 124513 (2005). The approximation $\gamma=A \epsilon a^{1-d}\left(1-T / T_{c}\right)^{3 / 2}$ with $A$ $=0.24\left(M / a^{d+2} k_{B} T\right)^{1 / 2}$ holds within a few percents in the range $0.6 \lesssim T / T_{c}<1$ for the van der Waals model. Here $M$ is assumed to be independent of $n$. For example, $\gamma=0.02 \epsilon \ell / v_{0}$ at $T / T_{c}=0.85$ in terms of $\ell$ in Eq. (3.1).

[47] L. D. Landau and E. M. Lifshitz, Fluid Mechanics (Pergamon, New York, 1959).

[48] J. W. Cahn, J. Chem. Phys. 66, 3667 (1977).

[49] From the argument of the entropy production rate only, our heat flux $\boldsymbol{J}_{e}$ in Eq. (2.39) and our diagonal pressure $p_{1}$ in Eq. (2.48) may be generalized to $\boldsymbol{J}_{e}^{\prime}=\boldsymbol{J}_{e}+\alpha \boldsymbol{J}_{\mathrm{GA}}$ and $p_{1}^{\prime}=p_{1}$ $+\alpha n \nabla n \cdot \nabla(M / T)$, where $\alpha$ is arbitrary. The expressions in Refs. [24,26] follow for $\alpha=1$. However, the arguments in Appendix A lead to $\alpha=0$.

[50] D. Bedeaux, Adv. Chem. Phys. 64, 47 (1986). Here a systematic derivation is given for the surface stress tensor and the surface boundary conditions.

[51] R. B. Bird, W. E. Stewart, and E. N. Lightfoot, Transport Phenomena (Wiley, New York, 2002), p. 272.

[52] A. Onuki, H. Hao, and R. A. Ferrell, Phys. Rev. A 41, 2256 (1990); A. Onuki and R. A. Ferrell, Physica A 164, 245 (1990).

[53] D. Dahl and M. R. Moldover, Phys. Rev. Lett. 27, 1421 (1971); J. S. Huang, W. I. Goldburg, and M. R. Moldover, ibid. 34, 639 (1975).

[54] P. Guenoun, B. Khalil, D. Beysens, Y. Garrabos, F. Kommoun, B. Le Neindre, and B. Zappoli, Phys. Rev. E 47, 1531 (1993); D. Beysens, Y. Garrabos, V. S. Nikolayev, C. Lecoutre-Chabot, J. P. Delville, and J. Hegseth, Europhys. Lett. 59, 245 (2002).

[55] Y. Miura, S. Yoshihara, M. Ohnishi, K. Honda, M. Matsumoto, J. Kawai, M. Ishikawa, H. Kobayashi, and A. Onuki, Phys. Rev. E 74, 010101(R) (2006).

[56] A. Furukawa and A. Onuki, Phys. Rev. E 66, 016302 (2002).

[57] D. H. Rothman and S. Zaleski, Rev. Mod. Phys. 66, 1417 (1994).

[58] B. J. Palmer and D. R. Rector, Phys. Rev. E 61, 5295 (2000).

[59] As a technique increasing the numerical stability, we defined $\boldsymbol{q} \equiv \lambda \nabla T$ on the midpoints $[i+1 / 2, j+1 / 2]$, where $[i, j]$ represent the lattice points. For example, $q_{x}$ at the middle point $[i$ $+1 / 2, j+1 / 2]$ was defined by $(\lambda[i+1, j+1]+\lambda[i, j+1]+\lambda[i$ $+1, j]+\lambda[i, j])(T[i+1, j+1]-T[i, j+1]+T[i+1, j]-T[i, j]) / 8$.

Then $\nabla_{x} \lambda \nabla_{x} T$ at the point $[i, j]$ was set equal to $\left(q_{x}[i+1 / 2, j\right.$ $+1 / 2]-q_{x}[i-1 / 2, j+1 / 2]+q_{x}[i+1 / 2, j-1 / 2]-q_{x}[i-1 / 2, j$ $-1 / 2]) / 2$. The same technique was also used for $\nabla_{m} \eta \nabla_{\ell} v_{k}$. 